\title{
Dual porosity modelling of the coupled mechanical response of coal to gas flow and adsorption
}

\author{
Min Chen, Lee J. Hosking, Richard J. Sandford, Hywel. R. Thomas \\ Geoenvironmental Research Centre, Cardiff School of Engineering, Cardiff University, Queen's Buildings, The
}

Parade, Newport Road, CF24 3AA, Cardiff, UK

\begin{abstract}
This paper presents the inclusion of explicit dual poroelastic mechanical behaviour as part of an existing dual porosity numerical model of multiphase, multicomponent chemical-gas transport. The dual poroelastic framework employed considers the pore structure changes occurring as a result of high pressure carbon dioxide injection into coal, particularly the adsorption-induced coal swelling that has been found to limit injectivity in field trials of carbon sequestration in coalbeds around the world. To address this issue, the surface stress of the fluid-solid interface is introduced into the constitutive relation for dual porosity effective stress in order to investigate the coal deformation and porosity changes related to adsorption behaviour. A new porosity model is presented, in which the impacts of gas flow and coal deformation are incorporated, and an interaction coefficient is proposed to explain the effect of fracture-matrix interactions on the porosity evolution. The model is verified and validated in this work against relevant analytical solutions and experimental results, and applied to study the gas flow behaviour and structural changes of coal. The results show that carbon dioxide injection not only causes coal swelling but also has the potential to change the internal pore structure of coal. The variation of fracture porosity is not monotonic as a competing result of effective stress and internal fracture-matrix interactions. However, the matrix porosity is found to increase during carbon dioxide injection, which seems to be a key contributor to the swelling phenomenon.
\end{abstract}

Keywords: carbon sequestration; dual poroelasticity; mechanical response; adsorption; gas flow

\section{Introduction}

Carbon sequestration in deep coal deposits has received considerable attention over the past two decades in particular, principally due to concerns over the atmospheric concentration of greenhouse gases and the commercial significance of coalbed methane around the world (Sampath et al., 2017; White et al., 2005). A number of field demonstration projects of carbon sequestration in coal have been conducted, the largest in terms of carbon dioxide $\left(\mathrm{CO}_{2}\right)$ injection having been for the enhanced recovery of coalbed methane at the Allison Unit in the San Juan Basin (USA), where it was found that $\mathrm{CO}_{2}$ injection reduced coal permeability, leading to a loss of injectivity (Reeves et al., 2003). Similar observations have been 
made in other pilot projects, such as the RECOPOL project in Poland (van Bergen et al., 2006), the Qinshui project in China (Wong et al., 2007), and the Yubari project in Japan (Fujioka et al., 2010). The drop of injectivity is attributed to $\mathrm{CO}_{2}$ adsorption-induced coal matrix swelling and the associated permeability reduction as the fracture flow pathways are compressed (Oudinot et al., 2011).

Overcoming the challenge of coal swelling is critical for the future prospects of carbon sequestration in coal and requires improvements to our understanding of the phenomenon at the basic level. To this end, this paper presents a new theoretical formulation for the coupled mechanical behaviour of coal as $\mathrm{CO}_{2}$ is injected. An existing dual porosity numerical model of multiphase, multicomponent chemical-gas transport, presented by Hosking et al. (2017), forms the basis of the current work. A fully coupled dual poroelastic model of gas transport and adsorption is developed theoretically and numerically to investigate the gas flow and mechanical behaviour of coal in this study. Specifically, the implicit approach to mechanical behaviour adopted by Hosking et al. is now described explicitly. As with the previous work, the new formulation is implemented in the coupled thermal, hydraulic, chemical, and mechanical (THCM) model, COMPASS, developed at the Geoenvironmental Research Centre by Thomas and co-workers (Hosking et al., 2017; Seetharam et al., 2007; Thomas and He, 1998; Thomas et al., 2012).

\section{Background}

The classical theory of poroelasticity developed by Biot is generally applied to account for the coupling interaction between fluid flow and deformation of the solids. The effective stress principle is one of the most fundamental contributions in this theory, which generally allows the application of the principle of continuum solid mechanics to the coupled flow-deformation models for the fluid-filled deformable porous media with a single dominant porosity (e.g. Detournay and Cheng, 1993). However, many natural geomaterials, such as coal, have two distinct porosities, which can be represented by equivalent homogeneous dual porosity systems (Warren and Root, 1963). To establish a fundamental link between mechanical effects and fluid flow, a rigorous evolution of effective stress for dual porosity media was derived, which has been widely used for hydro-mechanical analysis, especially for the flow of slightly compressible fluids without adsorption (Lewis and Pao, 2002). A similar expression for the effective stress in multi-phase porous media exhibiting two scales of porosity was derived by Borja and Koliji (2009) using the mixture theory to identify energy-conjugate variables. Nevertheless, such models fail to describe the flow-mechanical response of adsorptive dual porosity media.

Coal seams are naturally fractured reservoirs, with a macroporous interconnected fracture network providing the principal pathways for bulk fluid flow. The surrounding coal matrix contains micropores within which the majority gas adsorption occurs (Espinoza et al., 2014). Coal swells in the order of a few percent when it is exposed to $\mathrm{CO}_{2}$, methane $\left(\mathrm{CH}_{4}\right)$ and nitrogen $\left(\mathrm{N}_{2}\right)$ (Day et al., 2011; Pini et al., 2009). A Langmuir-type equation is most commonly used to theoretically describe the deformation of 
coal induced by the adsorption of gas. However, it cannot accurately predict total strain under high pressure. Experiments performed by Hol and Spiers (2012) show that the total strain increases monotonically with $\mathrm{CO}_{2}$ at pressures between 0 to $20 \mathrm{MPa}$, then decreases as the gas pressure keeps increasing (> $20 \mathrm{MPa}$ ). Recent thermodynamic models developed based on the theory of change in surface energy as a result of adsorption conceptualize surface tension or stress of the solid-fluid interface as a pre-stress, which can induce elastic strain even under stress-free conditions (Nikoosokhan et al., 2012; Pan and Connell, 2007; Vandamme et al., 2010). Different from the Langmuir-type equation, thermodynamic models attribute coal volumetric changes to two processes, namely, coal compression due to pressure and swelling due to gas adsorption. Importantly, the phenomenon that swelling strain may decrease after reaching a swelling maximum can be described.

The adsorption-induced swelling and desorption-induced shrinkage have a considerable impact on the changes in porosity and permeability of coal. In order to predict the variation of permeability, a number of permeability models have been developed. Although these permeability models can match the history data well, the emphasis of these models is laid on the change in fracture porosity; the effects of changes in the microporous coal matrix are ignored and the fundamental mechanism of permeability or porosity variation during coal expansion is not revealed. More recently, the concept of an internal swelling coefficient has been introduced to quantify the contribution of adsorption-induced matrix deformation to fracture aperture and coal permeability (e.g. Zhou et al., 2016). However, the physical meaning of internal swelling is assumed and so far not certain; the interaction between fractures, micropores and the solid skeleton is still an unsolved issue.

Carbon sequestration leads to a mechanical, physical and chemical response in coal seams. In this study, the theoretical and numerical developments of a fully-coupled model incorporating coal deformation and gas flow and adsorption are presented within the framework of poroelastic theory. The concept of surface stress is introduced into the effective stress for adsorptive dual porosity media to evaluate the adsorption-induced deformation. This adsorption-induced swelling model is coupled with a porosity model and a new permeability model is developed taking the fracture-matrix interaction into consideration. To allow the developed model to be applied with confidence, verifications of the model and evaluations against experimental results are presented. As mentioned previously, the new developments are implemented into the existing coupled thermal, hydraulic, chemical, and mechanical (THCM) model, COMPASS. The non-equilibrium flow and dynamic porosity and permeability evolution are investigated and discussed in this work.

\section{Theoretical formulation and numerical method}

Coal is a typical fractured rock consisting of the coal matrix surrounded by intersecting natural fractures or cleats, as shown in Fig. 1. Most coal seams have at least two regular cleat types, i.e. more continuous 
face cleats and less continuous butt cleats, which form a well-developed cleat system/natural fracture network and provide the primary pathways for fluid flow. In this paper, the coal structure is conceptualized using a dual continuum model, as depicted in Fig. 2. It is assumed that bulk fluid flow takes place only within the fracture continuum and the matrix continuum acts as a sink-source for fluid flow within the fracture continuum. By handling the fracture system and porous matrix as distinct continua over the domain, each has its own flow variable; namely, the gas concentrations in the fracture continuum $\left(c_{f}\right)$ and porous matrix continuum $\left(c_{m}\right)$ (Hosking et al., 2017). Mechanical behaviour is considered with the displacements $(\mathbf{u})$ used as the primary variable for deformation. Below, a set of governing equations for gas transport and fully-coupled coal deformation are developed based on the following assumptions: 1) coal is considered to be a dry, homogeneous, isotropic and dual poroelastic medium; 2) the strain is small; 3) the coal is saturated by ideal gas; 4) the coal is isothermal; 5) gas adsorption only occurs in the coal matrix; 6) gas flow in fractures satisfies Darcy's law.

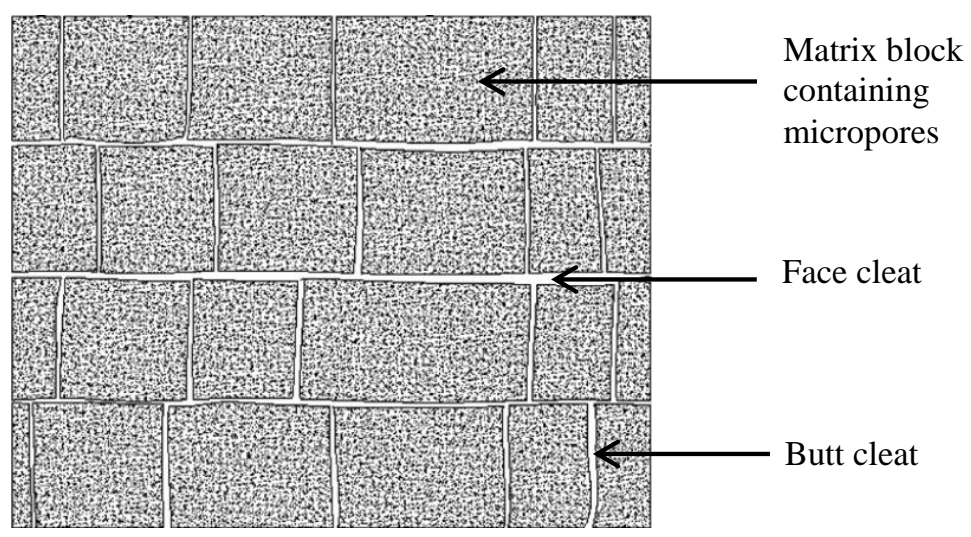

Fig.1 A schematic of naturally fractured coal (adapted from Shi and Durucan 2005b).

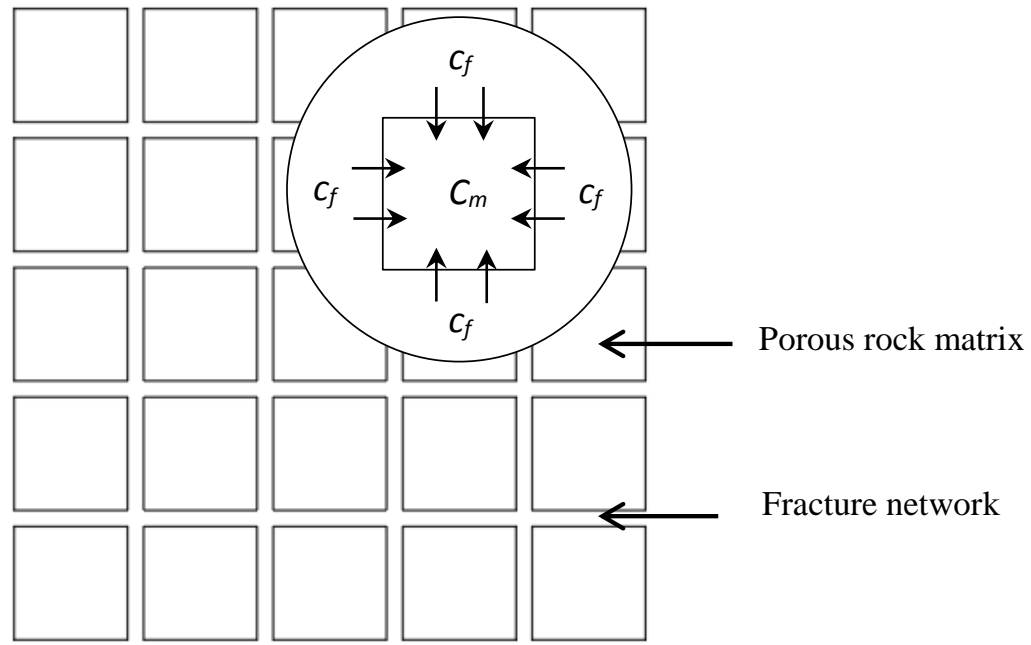

Fig. 2 Schematic of the conceptual geometry used to develop a dual porosity model and show the flow rate from the fracture network to the porous rock matrix. 


\subsection{Gas transport}

When gas is injected into coal, it flows mainly through the fracture network with a leak off into the porous coal matrix. For a dual porosity medium, two mass-balance equations are required to fully describe the gas flow behaviours. Following the work of Hosking et al. (2017), the governing equation for the migration of the gas in each continuum is expressed as $(\alpha=f$ refers to fracture network and $\alpha=m$ refers to porous matrix)

$$
\frac{\partial}{\partial t}\left(c_{\alpha} \theta_{\alpha}\right)+R_{\alpha}=-\nabla \cdot\left(c_{\alpha} \mathbf{v}_{\alpha}\right)+\gamma Q_{e \alpha}
$$

where $\theta_{\alpha}$ is the gas content, $R_{\alpha}$ is the sink-source term for the accumulation-generation of gas due to adsorption per unit volume of medium, $\mathrm{Q}_{\mathrm{e} \alpha}$ is the gas exchange rate between coal matrix and fracture, with $\gamma=1$ if $\alpha=f$ or $\gamma=-1$ if $\alpha=m$, and $\mathbf{v}_{\alpha}$ is the gas velocity. Due to the assumption that coal is only saturated with gas, the gas content equals to the porosity of coal, i.e.

$$
\theta_{\alpha}=n_{\alpha}
$$

The relative gas velocity is defined by Darcy's law as

$$
\mathbf{v}_{\alpha}=-\frac{k_{\alpha}}{\mu} \nabla u_{g \alpha}
$$

where $k_{\alpha}$ is fracture permeability of $\alpha$ continuum, $\mu$ is the viscosity of the gas, and $u_{g \alpha}$ is the gas pressure. The effect of gravity is neglected. Application of the ideal gas law allows the gas pressure and density to be expressed in terms of gas concentration, giving

$$
u_{g \alpha}=R T c_{\alpha}
$$

where $R$ is the universal gas constant and $T$ is the temperature.

The gas transfer from the fracture into matrix is assumed to be driven by the difference of concentration between the matrix and fracture, therefore, the gas exchange rate can be written as

$$
Q_{e \alpha}=D a\left(c_{m}-c_{f}\right)
$$

where $a$ is the shape factor of the matrix and $D$ is the gas diffusion coefficient. Generally a diffusion time, $\tau$, is introduced to approximate the diffusivity of the coal matrix block (Liu et al., 2015)

$$
\tau=\frac{1}{D a}
$$

The amount of gas adsorbed in coal is generally described with a Langmuir isotherm as a function of gas pressure (Hosking et al., 2017). Taking this approach in this work, the amount of adsorbed gas per unit volume of medium is described as 


$$
R_{\alpha}=\rho_{c} V_{s} \frac{\partial S}{\partial t}
$$

where $\rho_{c}$ is coal density, $V_{S}$ is mole volume at standard conditions, and $S$ is the adsorbed concentration, given by

$$
\mathrm{S}=\frac{V_{L} B u_{g m}}{B u_{g m}+1}
$$

where $\mathrm{V}_{\mathrm{L}}$ is the Langmuir volume constant and $B$ is the Langmuir pressure constant.

According to the aforementioned assumptions, there is no adsorbed phase in the fracture network, i.e. $R_{f}=0$. Substitution of equations (2)-(8) into equation (1) gives the governing equations for gas flow in a dual porosity medium

$$
\begin{aligned}
& n_{f} \frac{\partial c_{f}}{\partial t}+c_{f} \frac{\partial n_{f}}{\partial t}=\nabla \cdot\left(u_{g f} \frac{k_{f}}{\mu} \nabla c_{f}\right)+\frac{1}{\tau}\left(c_{m}-c_{f}\right) \\
& \left(n_{m}+V_{s} \rho_{c} \frac{\partial S}{\partial c_{m}}\right) \frac{\partial c_{m}}{\partial t}+C_{m} \frac{\partial n_{m}}{\partial t}=-\frac{1}{\tau}\left(c_{m}-c_{f}\right)
\end{aligned}
$$

\subsection{Coal deformation}

The deformation behaviour of coal is generally represented by the three basic principles of poroelastic theory, namely, the stress equilibrium, strain-displacement, and strain-stress relations. Following the traditional conventions, a comma followed by subscripts denotes the differential with respect to spatial coordinates and repeated indices denote a summation over the range of the indices. Stress equilibrium is expressed as (stress and strain are positive in tension, whereas fluid pressure is positive for compression in this study)

$$
\sigma_{i j, j}+F_{j}=0
$$

where $F_{j}$ is the component of body force vector.

The elastic stress-strain relation is

$$
\sigma_{i j}^{\prime}=2 G \varepsilon_{i j}+\lambda \varepsilon_{k k} \delta_{i j}
$$

The strain-displacement relation is

$$
\varepsilon_{i j}=\frac{1}{2}\left(u_{i, j}+u_{j, i}\right)
$$

where $\varepsilon_{i j}$ is the component of total stress tensor, $u_{i}$ is the component of solid displacement vector, $G=$ $E / 2(1+v)$ is the shear modulus, $\lambda=E v /(1+v)(1-2 v)$ is the Lamé constant, $E$ is Young's 
modulus, and $v$ is Poisson's ratio. $\varepsilon_{k k}=\varepsilon_{x x}+\varepsilon_{y y}+\varepsilon_{z z}$ is the volumetric strain of the bulk porous medium.

However, when the porous material interacts with adsorptive fluid, the deformation behaviour can no longer be described using the conventional stress-strain relation. Before giving the governing equation for coal deformation, it is necessary to represent the change in effectives stress induced by gas adsorption. In classic poroelastic theory, the surface energy effect is ignored. When surface energy is to be considered, it should be recognized that the saturated porous media not only contains the solid matrix and fracture system but also the solid-fluid interface. Part of the free energy of the system can be stored at this solid-fluid interface. In the surface science literature, in addition to surface energy quantifying the work of creating more surface in the undeformed material (e.g. creating a crack or a cavity in a solid) and related to the breaking of the interatomic bonds, the surface stress is introduced to quantify the work of stretching the existing surface through changing the distance at a constant number of atoms (Kramer and Weissmüller, 2007). This surface stress depends on the nature and on the state of fluid. All fluid molecules at the solid-fluid interface are not in their bulk state anymore, but are said to be adsorbed by interaction with the solid matrix and the solid surface is stretched if the surface is deformable. As a result, the surface stress is modified (Vandamme et al., 2010).

As mentioned above, the surface stress provides a basis for estimating the coal deformation related to adsorption behaviour. Before introducing surface stress into a constitutive equation derived from poroelastic mechanics, the surface stress must be transformed from the molecular level into macroscopic stress. Following the work of Nikoosokhan et al. (2012), the surface stress of the solidfluid interface is conceptualized as two pre-pressures and applied to the standard constitutive relation, which establishes a fundamental link between the adsorption characteristics of the solid-fluid interface and the mechanical response of the porous medium. One pre-pressure acts in the fracture system and the other acts in the coal matrix. Within this context, the effective stress in incremental form for a fractured porous medium saturated with adsorptive gases is expressed as

$$
d \sigma_{i j}^{\prime}=d \sigma_{i j}+b_{f} d\left(u_{g f}-u_{f}^{s}\right) \delta_{i j}+b_{m} d\left(u_{g m}-u_{m}^{s}\right) \delta_{i j}+d u_{f}^{s} \delta_{i j}
$$

where $\sigma_{i j}^{\prime}$ is the component of effective stress tensor, $\sigma_{i j}$ is the component of total external stress tensor, $\delta_{i j}$ is Kronecker's delta tensor $\left(\delta_{\mathrm{ij}}=1\right.$ for $\mathrm{i}=\mathrm{j}, \delta_{\mathrm{ij}}=0$ for $\left.\mathrm{i} \neq \mathrm{j}\right)$, and $b_{f}$ and $b_{m}$ are the effective stress coefficients for fracture pressure and matrix pressure, respectively, defined as

$$
\begin{gathered}
b_{f}=1-\frac{K}{K_{m}} \\
b_{m}=\frac{K}{K_{m}}-\frac{K}{K_{s}}
\end{gathered}
$$


where $K=E / 3(1-2 v)$ is the bulk modulus, $E$ is the Young's modulus, and $K_{m}=E_{m} / 3(1-2 v)$ is the modulus of coal matrix with $E_{m}$ being Young's modulus of the coal matrix, which can be obtained from the experiments performed on specimens an order of magnitude larger than the spacing of the matrix pores but devoid of fractures. $K_{S}$ is the modulus of the solid constituent and usually cannot be measured directly; however, it can be determined through matching the experimental measurement, or by using the equation (Liu et al., 2015)

$$
K_{s}=\frac{K_{m}}{1-3 n_{m}(1-v) /[2(1-2 v)]}
$$

The pre-pressure $u_{f}^{S}$ and pre-pressure $u_{m}^{s}$ are related to the surface stress

$$
\begin{aligned}
& u_{f}^{s}=\xi_{f} \Delta \sigma^{s} \\
& u_{m}^{s}=\xi_{m} \Delta \sigma^{s}
\end{aligned}
$$

where $\xi_{f}$ and $\xi_{m}$ are two constant material parameters representing the correlations between changes in the adsorption area of the matrix pore and in the volumetric strain and porosity of the matrix (Nikoosokhan et al., 2013). $\Delta \sigma^{S}$ is the change in surface stress due to adsorption. For the case of a fluid mixture, the change in surface stress can be obtained according to the Gibbs-Duhem relation (Nikoosokhan et al., 2012)

$$
\Delta \sigma^{s}=-\int_{u_{g 0}}^{u_{g}} \Gamma \bar{V}_{b} d u_{g}
$$

where $\Gamma$ is the number of moles of fluid molecules adsorbed per unit area of the fluid-solid interface, and $\bar{V}_{b}=R T / u_{g}$ is the molar volume of the bulk fluid. If the fluid adsorption obeys a Langmuir isotherm, the change of surface stress is written as

$$
\Delta \sigma^{s}=-\Gamma^{\max } R T \ln \left(1+B u_{g}\right)
$$

where $\Gamma^{\max }$ is the Langmuir adsorption constant, representing the adsorption capacity of fluid per unit adsorption surface.

Combining equations (11)-(13) and using the effective stress given in equation (14) leads to the governing equation in incremental form for the deformation of a dual porosity medium saturated by adsorptive gas

$$
\boldsymbol{P D} \boldsymbol{P}^{\boldsymbol{T}} d \mathbf{u}+\boldsymbol{P} \mathbf{I}\left[b_{f} d u_{f}^{a}+b_{m} d u_{m}^{a}+d u_{f}^{S}\right]+d \boldsymbol{F}=\mathbf{0}
$$

where $u_{f}^{a}=u_{g f}-u_{f}^{s}$ and $u_{m}^{a}=u_{m}-u_{m}^{s}, \boldsymbol{P}$ is the strain matrix, and $\boldsymbol{D}$ is the elasticity matrix.

\subsection{Coupling deformation with dual porosity gas transport}

For a dual-porosity medium, the porosity of each continuum can be defined as 


$$
n_{\alpha}=\frac{V_{\alpha}}{V}
$$

where $V$ is the bulk volume and $V_{\alpha}$ is the pore volume of each continuum.

Thus, the porosity change of a deforming coal mass can be expressed as

$$
d n_{\alpha}=d\left(\frac{V_{\alpha}}{V}\right)=\frac{d V_{\alpha}}{V}-n_{\alpha} \frac{d V}{V}
$$

In the above two equations, $\frac{d V_{\alpha}}{V}$ represents the change in volume of the fracture and matrix pores over the current volume of the porous medium with respect to the moving solid. It is shown that the pore volume change per unit of bulk volume in a fractured porous medium is due to three components (Lewis and Pao, 2002). Firstly, the change in pore volume varies with the change in overall bulk volume of the porous medium. Secondly, the compression of the solid phase by fluid pressure occupying the matrix pores or fractures can affect the pore volume. Thirdly, matrix-fracture interaction due to the pressure difference between the pore regions can also contribute to changes in the respective pore volumes.

Gas adsorption not only deforms coal but also changes its internal structure. The fracture volume is strongly affected by the coal matrix swelling (Zhou et al., 2016). The pore fraction, defined as the ratio between the pore volume occupied by the subdomain in relation to the total volume of the pores in the entire porous media, is identified to be conjugated with the pore pressure difference by Borja (2009) using a thermodynamic approach; in other words, the pore fraction is a function of pore pressure difference. Adsorption in microporous adsorbents can be treated as a particular case of solution thermodynamics (Myers and Prausnitz, 1965); therefore, the matrix-fracture interaction due to adsorption can also be described with the help of the pore pressure difference between the fracture and matrix. On the other hand, if one of the fluid-pressure fields vanishes (e.g. fracture closure) or if the two fluid-pressure fields are equalized, the mechanical responses of a dual porosity system can reduce to the corresponding expressions of a single porosity system. Therefore, functional form of the pore volume of a single porosity system is maintained for a dual porosity system except for the term representing the internal interaction. Different from the work of Pao and Lewis (2002), in this work a new fracture-matrix interaction coefficient is introduced to represent the fracture-matrix interaction of porous media immersed in adsorptive fluid. The change in volume of fracture and matrix pores is expressed as

$$
\begin{aligned}
& \frac{d V_{f}}{V}=b_{f} d \varepsilon_{v}+a_{f} d u_{f}^{a}-\chi d u_{m f} \\
& \frac{d V_{m}}{V}=b_{m} d \varepsilon_{v}+a_{m} d u_{m}^{a}+\chi d u_{m f}
\end{aligned}
$$

where $u_{m f}=u_{m}^{a}-u_{f}^{a}, a_{f}=\left(b_{f}-n_{f}\right) / K_{s}, a_{m}=\left(b_{m}-n_{m}\right) / K_{s}$, and $\chi$ is the coefficient representing matrix-fracture interaction. This interaction coefficient is not necessarily constant but is a 
function of the pressure difference between fracture and matrix. According to Liu el al (2011c), the dynamic interaction between matrix and fracture is achieved through the transition of coal matrix swelling from local swelling to macro swelling. During local swelling, the coal deformation occurs under the constrained boundary condition and the fracture is easier to compress from the initial state; the interaction between the fracture and matrix is significant; namely, the interaction coefficient is relatively larger while the pressure difference between the fracture and matrix is small. However, when coal reaches a state of global swelling, it deforms under stress boundary and the pressure difference is large while the internal deformation becomes weak or even vanishes; namely, the interaction coefficient is relatively smaller. At this stage, the pore pressure plays a more important role in porosity change. Here it is proposed that there is an exponential relation between the interaction coefficient and pore pressure difference

$$
\chi=\chi_{0}\left\{1+\exp \left[-\omega\left(u_{m f}-u_{m f 0}\right)\right]\right\}
$$

where $\chi_{0}$ is the interaction coefficient at initial pressure difference $u_{m f 0}$, and $\omega$ is the interaction change rate. These two parameters are difficult to be determined directly through experimental treatment. In this work, these two parameters can be determined by matching values of permeability while varying only the pressure and then choosing values for interaction coefficient and interaction change that best fit the measured data.

Considering small strain, $\frac{d V}{V}$ can be approximated as the increment of volumetric strain

$$
\frac{d V}{V} \approx \frac{d V}{V_{0}}=d \varepsilon_{v}
$$

Substitution of equations (23) and (24) into equation (22) yields

$$
\begin{aligned}
& d n_{f}=\left(b_{f}-n_{f}\right) d \varepsilon_{v}+a_{f} d u_{f}^{a}-\chi d u_{m f} \\
& d n_{m}=\left(b_{m}-n_{m}\right) d \varepsilon_{v}+a_{m} d p_{m}^{a}+\chi d u_{m f}
\end{aligned}
$$

It worth noting that even in cases where the solid constituent is incompressible and the bulk volume is constrained, there is still a pore volume change due to the third term. Also, equation (27) can reduce to the equation of a single porosity system if one of the fluid pressure fields does not exist, or if both fluid pressures reach equilibrium, which is consistent with the framework of poroelastic theory. Equation (26) provides a capacity to analyse the fracture-matrix interaction. Introducing the approximations $\frac{d()}{d t} \approx \frac{\partial O}{\partial t}$ yields the partial derivative of porosities with respect to time, as

$$
\begin{gathered}
\frac{\partial n_{f}}{\partial t}=\left(b_{f}-n_{f}\right) \frac{\partial \varepsilon_{v}}{\partial t}+a_{f} \frac{\partial u_{f}^{a}}{\partial t}-\chi\left(\frac{\partial u_{m}^{a}}{\partial t}-\frac{\partial u_{f}^{a}}{\partial t}\right) \\
\frac{\partial n_{m}}{\partial t}=\left(b_{m}-n_{m}\right) \frac{\partial \varepsilon_{v}}{\partial t}+a_{m} \frac{\partial u_{m}^{a}}{\partial t}-\chi\left(\frac{\partial u_{f}^{a}}{\partial t}-\frac{\partial u_{m}^{a}}{\partial t}\right)
\end{gathered}
$$


The permeability varies with porosity, which can be described by the widely used cubic relationship between permeability and porosity (e.g.Cui and Bustin, 2005), given as

$$
\frac{k_{f}}{k_{f 0}}=\left(\frac{n_{f}}{n_{f 0}}\right)^{3}
$$

where $k_{f 0}$ is the initial permeability of the fracture system and $n_{f 0}$ is the initial fracture porosity, respectively.

\subsection{Coupled governing equations}

Combining equations (9), (10) and (28) and rearranging produces the final equations for gas transport in a deformable dual porosity medium, including the effects of sorption induced swelling. For the fracture continuum

$$
C_{f f} \frac{\partial c_{f}}{\partial t}+C_{f m} \frac{\partial c_{m}}{\partial t}+C_{f u} \frac{\partial \mathbf{u}}{\partial t}=\nabla \cdot\left(K_{f f} \nabla c_{f}\right)+K_{f f_{-} f} c_{f}+K_{f f_{-m}} c_{m}
$$

in which

$$
\begin{aligned}
& C_{f f}=n_{f}+u_{g f}\left(a_{f}+\chi\right) \\
& C_{f m}=-\left[\frac{a_{f} \varphi_{f}-\chi\left(\varphi_{m}-\varphi_{f}\right)}{1+B u_{g m}} B R T-\chi\right] u_{g f} \\
& C_{f u}=c_{f}\left(b_{f}-n_{f}\right) \mathbf{I}^{T} \boldsymbol{P} \\
& K_{f f}=\frac{k_{f}}{\mu} R T c_{f} \\
& K_{f f_{-} f}=-\frac{1}{\tau} \\
& K_{f f_{-} m}=\frac{1}{\tau}
\end{aligned}
$$

where

$$
\varphi_{f}=\xi_{f} \Gamma^{\max }, \varphi_{m}=\xi_{m} \Gamma^{\max }
$$

For the matrix continuum

$$
C_{m m} \frac{\partial c_{m}}{\partial t}-C_{m f} \frac{\partial c_{f}}{\partial t}+C_{m u} \frac{\partial \mathbf{u}}{\partial t}=K_{m m_{-f}} c_{f}+K_{m m_{-} m} c_{m}
$$

in which

$$
C_{m m}=n_{m}+u_{g m}\left[a_{m}+\chi+\frac{a_{m} \varphi_{m}+\chi\left(\varphi_{m}-\varphi_{f}\right)}{1+B u_{g m}} B R T\right]+\mathrm{V}_{\mathrm{s}} \rho_{\mathrm{c}} \frac{\partial S}{\partial C_{m}}
$$




$$
\begin{aligned}
& C_{m f}=-\chi u_{g m} \\
& C_{m u}=c_{m}\left(b_{m}-n_{m}\right) \mathbf{I}^{T} \boldsymbol{P} \\
& K_{m m_{-} f}=\frac{1}{\tau} \\
& K_{m m \_m}=-\frac{1}{\tau}
\end{aligned}
$$

Terms on the right side of equations (31a) and (33a) represents the gas volume change per unit change in gas concentration and per unit volume of coal material. The first terms in equations (31a) and (33a) represent the volume of gas released or stored in the free phase. The second terms of equations (31a) and (33a) represent the gas volume change due to change in volume of fracture and matrix pores under the combined action of gas pressure and adsorption stress. The third term in equation (33a) represents the gas volume change in matrix pores due to adsorption or desorption. It is worth noting that the coefficients in equations (31b) and (33b) are coupling terms, showing the effect of gas concentration in one continuum on that in the other with the exception of the mass exchange between fracture and matrix. The interaction between the rate change in the volumetric strain and gas concentration in each continuum can be observed in equations (31c) and (33c).

To show the coupling between coal deformation and gas transport, the governing equation for coal deformation is re-written by replacing the gas pressure with gas concentration

$$
C_{u u} d \mathbf{u}+C_{u f} d c_{f}+C_{u m} d c_{m}+d \boldsymbol{F}=\mathbf{0}
$$

where

$$
\begin{aligned}
& C_{u u}=\boldsymbol{P} \mathbf{D} \boldsymbol{P}^{\boldsymbol{T}} \\
& C_{u f}=\boldsymbol{P} \mathbf{I} b_{f} R T \\
& C_{u m}=\boldsymbol{P} \mathbf{I}\left[\left(\varphi_{f} b_{f}+\varphi_{m} b_{m}-\varphi_{f}\right) \frac{B R^{2} T^{2}}{1+B R T c_{m}}+b_{m} R T\right]
\end{aligned}
$$

The gas pressure and adsorption stress in the deformation equation can be obtained through solving the equations for gas flow. Equations (29), (31) and (33) define a double porosity model that fully couples the stress-strain-sorption-flow behaviour of coal exposed to adsorbing gas. The cross-coupling relations of multi-physical fields are shown in Fig. 3. 


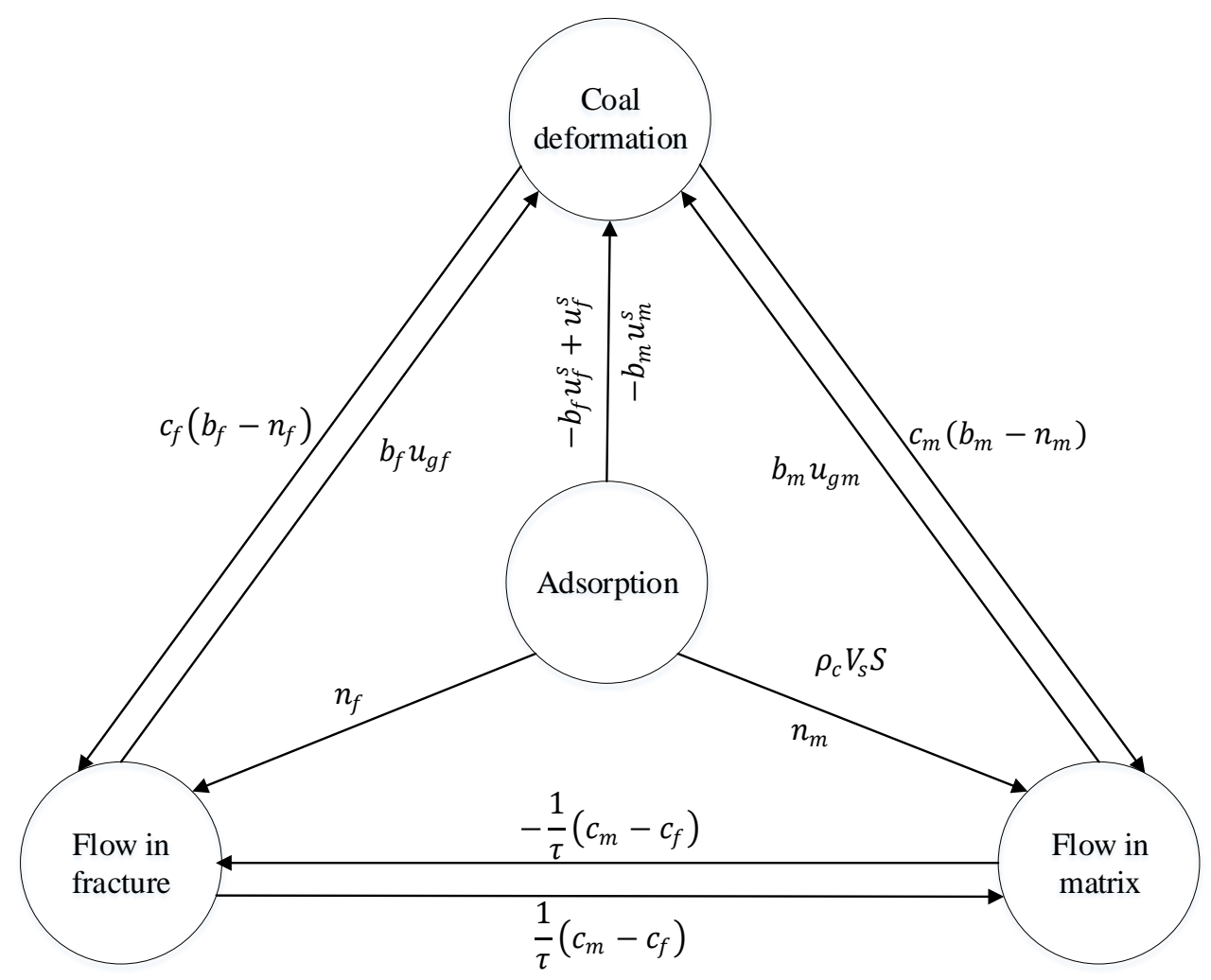

Fig.3 Schematic of cross-coupling relations among deformation, gas adsorption and flow behaviour.

\subsection{Numerical method}

The coupled gas flow-adsorption-deformation model presented in this paper has been implemented within the framework of the numerical model, COMPASS (COde of Modelling PArtially Saturated Soils), in which a Galerkin weighted residual method is employed to spatially discretize the governing equations. For temporal discretization, an implicit mid-interval backward difference time-stepping algorithm is employed. This has been found as a suitable solution for the highly non-linear class of equations such as the current application problem (Thomas and He, 1998).

Different from traditional flow simulators, which assign initial porosity and permeability at the start of the computation that then remain constant throughout the simulation, for coupled flow and deformation, porosity and permeability need to update each time step. The loose coupling algorithm is employed for temporal discretization of porosity. This algorithm is described in detail by Minkoff et al. (2003). Each time step first involves solving the conservative transport and deformation equations to calculate the gas concentration and strain and ultimately the porosity and permeability are updated.

\section{Model verification and validation}

In order to examine the correctness of the numerical implementation of the enhanced theoretical and numerical developments implemented in the model, a set of verification and validation tests have been 
performed. Verification test are employed to ensure that the solution of the model is accurate, whilst the validation tests are performed to ensure the underlying theory can accurately reflect the material behaviour and related processes. Several relevant steady and transient analytical solutions are taken as benchmarks for verification testing, whilst laboratory results are used for validation testing. Considering that a unified analytical solution for gas flow in dual porous media is impractical or impossible to find under the conditions of adsorption and deformation considered in this work, all tests considering an analytical solution as a benchmark are based on a single porosity medium. These tests include: (1) transient gas flow in an infinite radial system with a constant source; (2) transient gas flow in an infinite radial system with adsorption; (3) adsorption-induced deformation; (4) testing the permeability model.

\subsection{Transient flow with and without adsorption}

In this section, two verification tests are performed for gas flow with or without adsorption in an infinite radial system. For an isothermal single porosity system, the gas flow in porous media with adsorption is expressed as

$$
\frac{\partial\left(n c+V_{s} \rho_{c} S\right)}{\partial t}=-\nabla \cdot(c \mathbf{v})
$$

The problem concerns transient gas flow across a 1-D radial uniform region. A constant gas mass injection rate is imposed at a well and the initial pressure is uniform throughout the overall region. The well boundary condition is a constant gas injection rate of $1 \times 10^{-5} \mathrm{~kg} \mathrm{~s}^{-1}$. The parameters required for the verification test are listed in Table 1.

Table1 Parameters for model test

\begin{tabular}{|l|l|}
\hline Parameters & Value \\
\hline Porosity, $n$ & 0.3 \\
\hline Permeability, $k, \mathrm{~m}^{-2}$ & $1.0 \mathrm{e}^{-15}$ \\
\hline Mole volume, $V_{s}, \mathrm{~mol} \mathrm{~m}{ }^{-3}$ & 43.1 \\
\hline Gas viscosity, $\mu, \mathrm{Pa} \cdot \mathrm{s}$ & $1.84 \mathrm{e}^{-5}$ \\
\hline Initial pressure, $\mathrm{Pa}$ & $1.0 \mathrm{e}^{5}$ \\
\hline Thickness, $\mathrm{m}$ & 1.0 \\
\hline Langmuir volume constant, $V_{L}, \mathrm{~m}^{3} \mathrm{~kg}^{-3}$ & 0.015 \\
\hline Langmuir pressure constant, $B, \mathrm{~Pa}^{-1}$ & $1.0 \mathrm{e}^{-6}$ \\
\hline Density of material, $\rho_{c}, \mathrm{~kg} \mathrm{~m}^{-3}$ & 1250 \\
\hline
\end{tabular}

The approximate analytical solutions and the details about the analytical solution derivation are included in work of $\mathrm{Wu}$ et al. (2014). Only the verification results are shown here. Fig. 4 compares the results of pressure distribution obtained from the analytical solution and numerical simulation at 1 day. 
Two situations, with and without adsorption, are considered. The numerical solutions are shown with the analytical solutions. It is noted that the change in gas presssure in the test involving adsorption is slower, which is the expected result since adsorption decreases the amount of free gas and retards flow.

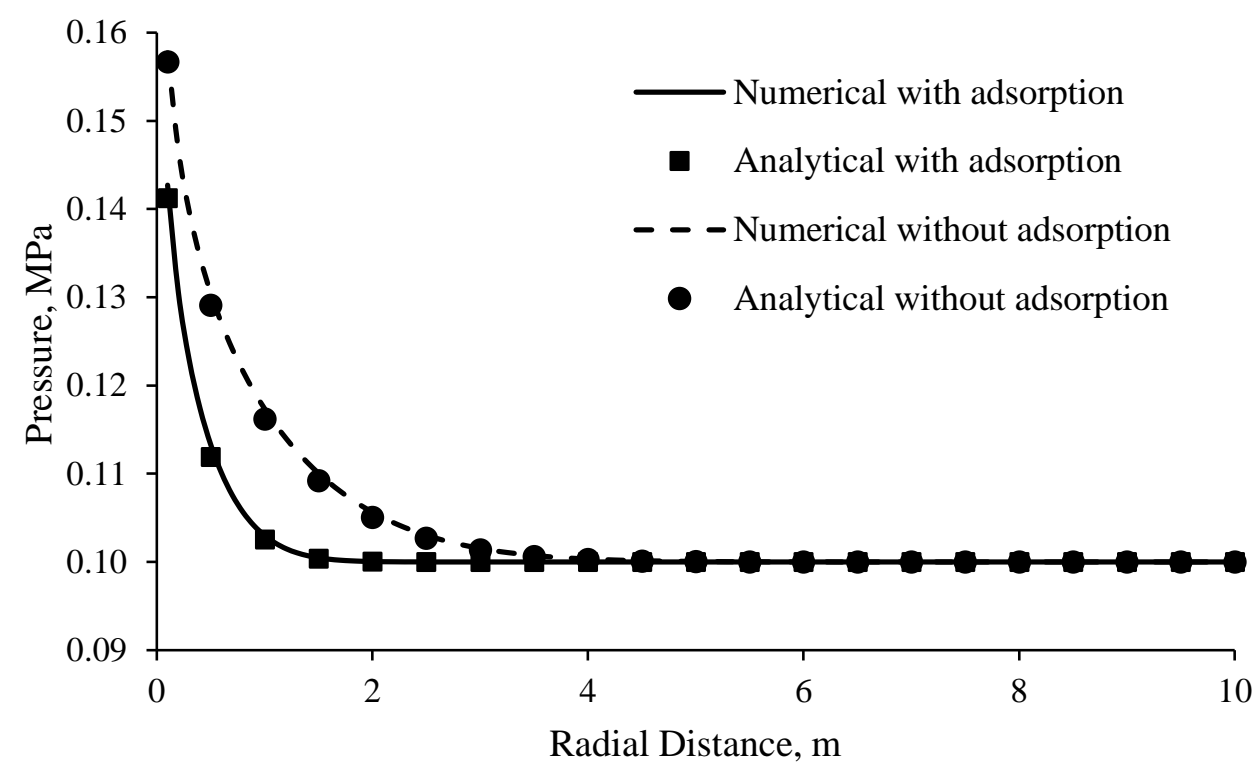

Fig. 4 Comparison of gas pressure profiles with and without adsorption calculated using the numerical and the analytical solutions in a radially infinite system at 1 day.

\subsection{Deformation with adsorption compared to experimental data}

This section examines the performance of the constitutive relationship for deformation involving gas adsorption behaviour. Two sets of experimental data from core Anderson 01 (Robertson, 2005) and core No. 01 (Pan et al., 2010) were used to evaluate the developed deformation model. If the fluid adsorption isotherm is determined, adding the three principle strains given by equation (12) leads to a relation between mean stress $\bar{\sigma}$ and volumetric strain $\varepsilon_{v}$

$$
\varepsilon_{v}=\frac{\bar{\sigma}}{K}
$$

where

$$
\bar{\sigma}=\left(\sigma_{11}+\sigma_{22}+\sigma_{33}\right) / 3+b_{f} u_{g f}+b_{m} u_{g m}-\left(\xi_{f} b_{f}+\xi_{m} b_{m}-\xi_{f}\right) \Delta \sigma^{s}
$$

Coal is comprised of the cleat macroporosity and coal matrix microporosity. Swelling and shrinkage (volumetric change) of coal during adsorption and desorption of gas (like $\mathrm{CO}_{2}, \mathrm{CH}_{4}$ ) is a well-known phenomenon. Robertson (2005) carried out a series of tests on longitudinal strain of subbituminous coal collected from the Anderson coal seams using pure gas and mixed gases $\left(\mathrm{N}_{2} 51 \%\right.$ and $\left.\mathrm{CO}_{2} 49 \%\right)$. Pan et al. (2010) measured the $\mathrm{CH}_{4}$ and $\mathrm{CO}_{2}$ adsorption-induced swelling strain of bituminous coal. The experimental results are used here to test the performance of the effective stress model proposed. It is assumed that the pressures of the fluid in the cleat and in the pores are identical at equilibrium $\left(u_{g f}=\right.$ 
$u_{g m}=u_{g}$ ) and the adsorption of pure gas obeys the Langmuir isotherm. Based on the experimental conditions $\left(\sigma=-u_{g}\right)$, the change in the surface stress is based on the assumption of an ideal gas, rewritten as

$$
\bar{\sigma}=-u_{g}+b u_{g}+\pi R T \ln \left(1+B u_{g m}\right)
$$

where $\pi=\left(\xi_{f} b_{f}+\xi_{m} b_{m}-\xi_{f}\right) \Gamma_{\max }$, which can be viewed as a Langmuir constant for surface stress, representing the effect of change in coal structure on the adsorbed amount of gas. It can be obtained by matching the experimental measurements. $u_{g}$ is gas pressure at the equilibrium state and $b$ is the familiar Biot's coefficient.

For swelling induced by the adsorption of a binary gas mixture, with constant gas composition and changing total pressure (Robertson, 2005), the change in mean stress can be expressed as

$$
\bar{\sigma}=-u_{g}+b u_{g}+\frac{\pi_{C_{2} B_{C O}} \theta_{C O_{2}}+\pi_{N_{2}} B_{N_{2}} \theta_{N_{2}}}{B_{C O_{2}} \theta_{C O_{2}}+B_{N_{2}} \theta_{N_{2}}} R T \ln \left[1+\left(B_{C_{2}} \theta_{C O O_{2}}+B_{N_{2}} \theta_{N_{2}}\right) u_{g}\right]
$$

where $\theta_{\mathrm{CO}_{2}}$ and $\theta_{\mathrm{N}_{2}}$ are the gas fractions in the mixture.

The parameters required for the adsorption-induced swelling model can be determined by matching the experimental data of coal swelling. Parameters for the Anderson coal employed in Robertson's (2005) experiments and Pan et al.'s (2010) experiments are given in Table 2. The mechanical properties used for testing Roberson's experimental measurement are obtained based on the bulk modulus of coal matrix provided and the measured longitudinal strain of Anderson coal induced by various helium gas pressures at a constant temperature. The adsorption-related parameters are obtained by matching the experimental data. The parameters for validation against Pan et al.'s (2010) experiments are chosen from Chen et al. (2012), Connell et al. (2010) and Pan et al. (2010).

Fig. 5 and Fig. 6 show the comparison between the calculated response and the experimental data. The model results compare well with the measurement results for both pure $\left(\mathrm{CO}_{2}, \mathrm{CH}_{4}, \mathrm{~N}_{2}\right)$ and mixed gas $\left(\mathrm{N}_{2} 51 \%\right.$ and $\left.\mathrm{CO}_{2} 49 \%\right)$ in Robertson's (2005) experiments and pure gas $\left(\mathrm{CO}_{2}, \mathrm{CH}_{4}\right)$ in Pan et al.'s (2010) experiments, indicating the effective stress for adsorptive media with dual porosity is applicable to describe the deformation behaviour of porous media associated with adsorption. 
Table 2 Parameters for swelling model.

\begin{tabular}{|l|l|l|}
\hline \multicolumn{1}{|c|}{ Parameters } & $\begin{array}{c}\text { Robertson } \\
(\mathbf{2 0 0 5})\end{array}$ & $\begin{array}{l}\text { Pan et al. } \\
(\mathbf{2 0 1 0})\end{array}$ \\
\hline Bulk modulus of coal, $K, \mathrm{GPa}$ & 1.1 & 1.6 \\
\hline Bulk modulus of coal grains, $K_{S}, \mathrm{GPa}$ & 3.6 & 29.1 \\
\hline Bulk modulus of coal matrix, $K_{m}, \mathrm{GPa}$ & 2.8 & 21.3 \\
\hline Temperature, $T, \mathrm{~K}$ & 300 & 308 \\
\hline Langmuir constant, $\pi_{\mathrm{CO}_{2}}, \mathrm{~mol} \mathrm{~m}^{-3}$ & $1.6 \mathrm{e} 4$ & $1.17 \mathrm{e} 4$ \\
\hline Langmuir constant, $B_{\mathrm{CO}_{2}}, \mathrm{MPa}^{-1}$ & 0.88 & 0.19 \\
\hline Langmuir constant, $\pi_{C H_{4}}, \mathrm{~mol} \mathrm{~m}^{-3}$ & $4.3 \mathrm{e} 3$ & $6.04 \mathrm{e} 3$ \\
\hline Langmuir constant, $B_{C_{4}}, \mathrm{MPa}^{-1}$ & 0.67 & 0.34 \\
\hline Langmuir constant, $\pi_{N_{2}}, \mathrm{~mol} \mathrm{~m}^{-3}$ & $2.8 \mathrm{e} 3$ & - \\
\hline Langmuir constant, $B_{N_{2}}, \mathrm{MPa}^{-1}$ & 0.29 & - \\
\hline Biot's coefficient, $b$ & 0.7 & 0.945 \\
\hline
\end{tabular}

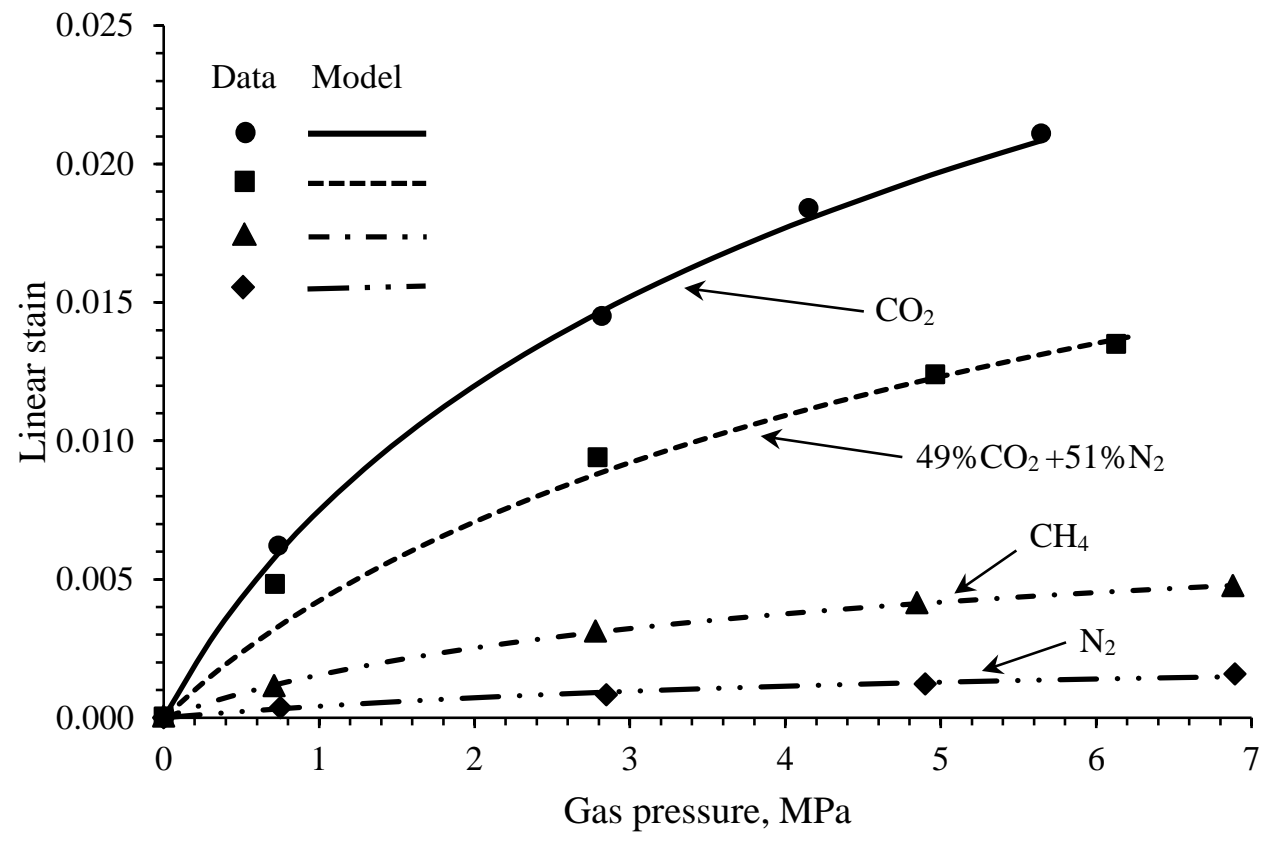

Fig. 5 Comparison between the experimental data and predicted swelling strain during the injection of $\mathrm{CO}_{2}, \mathrm{CH}_{4}, \mathrm{~N}_{2}$ and gas mixture of $49 \% \mathrm{CO}_{2}$ and $51 \% \mathrm{~N}_{2}$. 


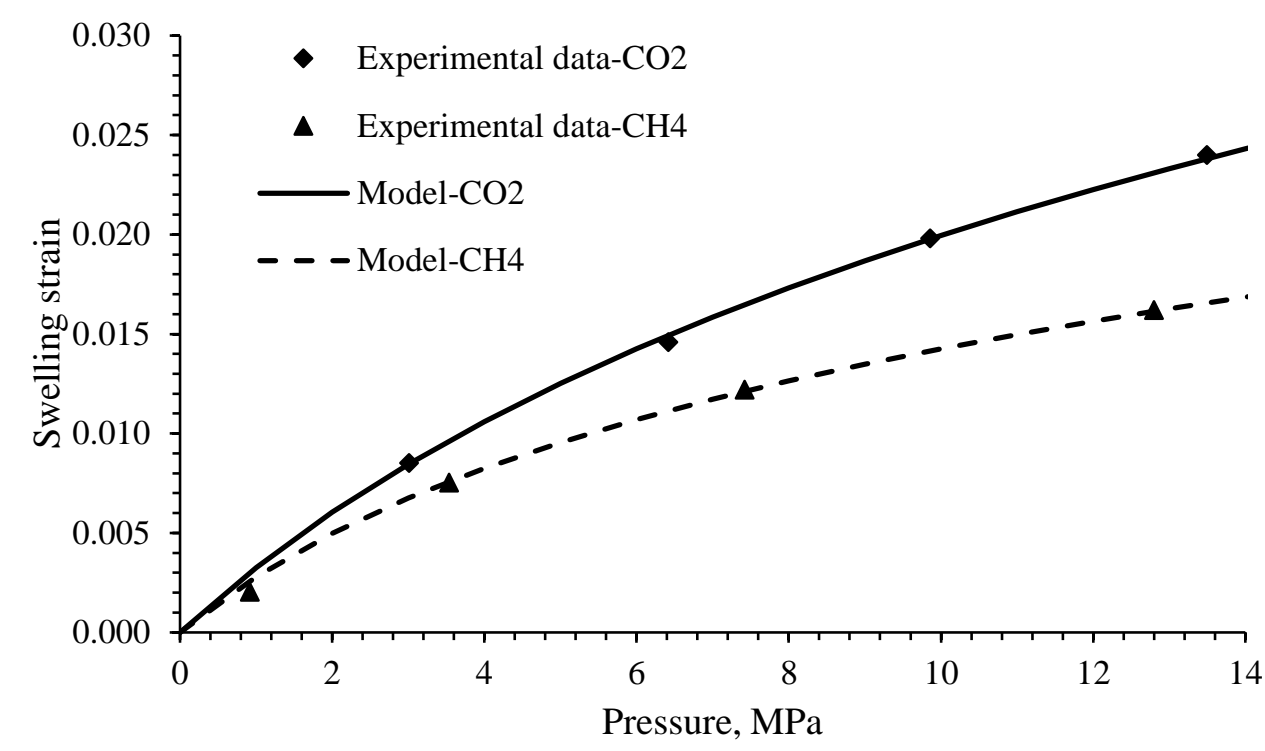

Fig. 6 Comparison of model predicted swelling strain with experimental data from Pan et al (2010).

\subsection{Permeability model compared to experimental data}

In this section, we examine the capacity of the newly-developed permeability model proposed in this work through a comparison with the published experimental observations by Robertson (2005) and Pan et al. (2010), which have been widely applied to evaluate permeability models (Chen et al., 2012; Connell et al., 2010; Liu and Rutqvist, 2010; Liu et al., 2011a; Liu et al., 2017). All permeability measurements $\left(\mathrm{CO}_{2}, \mathrm{CH}_{4}, \mathrm{~N}_{2}\right)$ were conducted at the final equilibrium stages under the conditions of free-swelling with constant confining stress in Robertson's experiments. Pan et al. (2010) conducted a series of permeability measurement of coal at constant pressure differences between confining stress and pore pressure.

Here the experimental data with $\mathrm{CO}_{2}$ and $\mathrm{CH}_{4}$ is used to evaluate the permeability model. Coal has two distinct pore structures, and the permeability of fracture or cleat networks is predominantly responsible for fluid flow in coal seams; the microporous matrix block has a negligible contribution to the coal permeability. Thus here only the facture porosity is taken into account. Inserting the equation (36) into equation (27a) gives

$$
d n_{f}=\frac{\left(b_{f}-n_{f}\right)}{K} d \bar{\sigma}+a_{f} d u_{f}^{a}-\chi d u_{m f}
$$

Generally the fracture porosity is small, and equation (39) can be integrated with time and substituted into equation (28), yielding

$$
\frac{k_{f}}{k_{f 0}}=\left(1+\frac{1}{n_{f 0}} \Delta \varepsilon_{f e}\right)^{3}
$$


where $\Delta \varepsilon_{f e}=\frac{b_{f}}{K}\left(\sigma^{\prime}-\sigma_{0}^{\prime}\right)+\frac{b_{f}}{K_{s}}\left(u_{f}^{a}-u_{f 0}^{a}\right)-\chi\left(u_{m f}-u_{m f 0}\right)$, similar to effective strain in Liu et al. (2011b), and $\sigma_{0}^{\prime}, u_{f 0}^{a}$ and $u_{m f 0}$ are the initial effective stress, fracture pressure, and pressure difference, respectively.

Equation (40) is used to fit the experimental measurements. The permeability data are collected from Robertson (2005) and Pan et al. (2010). Table 3 lists the parameters used. Some parameters used in this validation test are identical to those used in the previous deformation model validation. The surface stress coefficient, initial fracture-matrix interaction coefficient, and interaction change rate are obtained by matching the experimental results. The comparison results are presented in Figs. 7-9.

Table 3 Parameters used in permeability model test.

\begin{tabular}{|l|c|c|}
\hline \multicolumn{1}{|c|}{ Parameters } & $\begin{array}{c}\text { Robertson } \\
(\mathbf{2 0 0 5})\end{array}$ & $\begin{array}{c}\text { Pan et al. } \\
\mathbf{( 2 0 1 0})\end{array}$ \\
\hline Bulk modulus of coal, $K, \mathrm{GPa}$ & 1.1 & 1.6 \\
\hline Bulk modulus of coal grains, $K_{s}, \mathrm{GPa}$ & 3.6 & 29.1 \\
\hline Bulk modulus of coal matrix, $K_{m}, \mathrm{GPa}$ & 2.8 & 21.3 \\
\hline Temperature, $T, \mathrm{~K}$ & 300 & 308 \\
\hline Interaction change rate, $\omega, \mathrm{MPa}^{-1}$ & $7.4 \mathrm{e}-4$ & 0.0 \\
\hline Langmuir constant, $B_{C O_{2}}, \mathrm{MPa}^{-1}$ & 0.88 & 0.19 \\
\hline Biot's coefficient, $b_{f}$ & 0.6 & 0.925 \\
\hline Surface stress coefficient for $\mathrm{CO}_{2}, \varphi_{f}, \mathrm{~mol} \mathrm{~m}^{-3}$ & 0.0 & 0.0 \\
\hline Biot's coefficient, $b_{m}$ & 0.1 & 0.02 \\
\hline Surface stress coefficient for $\mathrm{CO}_{2}, \varphi_{m}, \mathrm{~mol} \mathrm{~m}^{-3}$ & $1.6 \mathrm{e} 5$ & $5.8 \mathrm{e} 5$ \\
\hline Initial interaction coefficient for $\mathrm{CO}_{2}, \chi_{0}, \mathrm{MPa}^{-1}$ & $3.5 \mathrm{e}-5$ & $6.9 \mathrm{e}-6$ \\
\hline Initial porosity, $n_{f 0} 0.013$ & 0.014 \\
\hline Langmuir constant, $B_{C H_{4}}, \mathrm{MPa}^{-1}$ & 0.013 & 0.34 \\
\hline Surface stress coefficient for $\mathrm{CH}_{4}, \varphi_{f}, \mathrm{~mol} \mathrm{~m}^{-3}$ & 0.0 & 0.0 \\
\hline Surface stress coefficient for $\mathrm{CH}_{4}, \varphi_{m}, \mathrm{~mol} \mathrm{~m}^{-3}$ & $4.3 \mathrm{e} 4$ & $3.0 \mathrm{e} 5$ \\
\hline Initial interaction coefficient $\mathrm{for}_{\mathrm{CH}} \mathrm{CH}_{4}, \chi_{0}, \mathrm{MPa}^{-1}$ & $1.1 \mathrm{e}-4$ & $1.2 \mathrm{e}-5$ \\
\hline
\end{tabular}




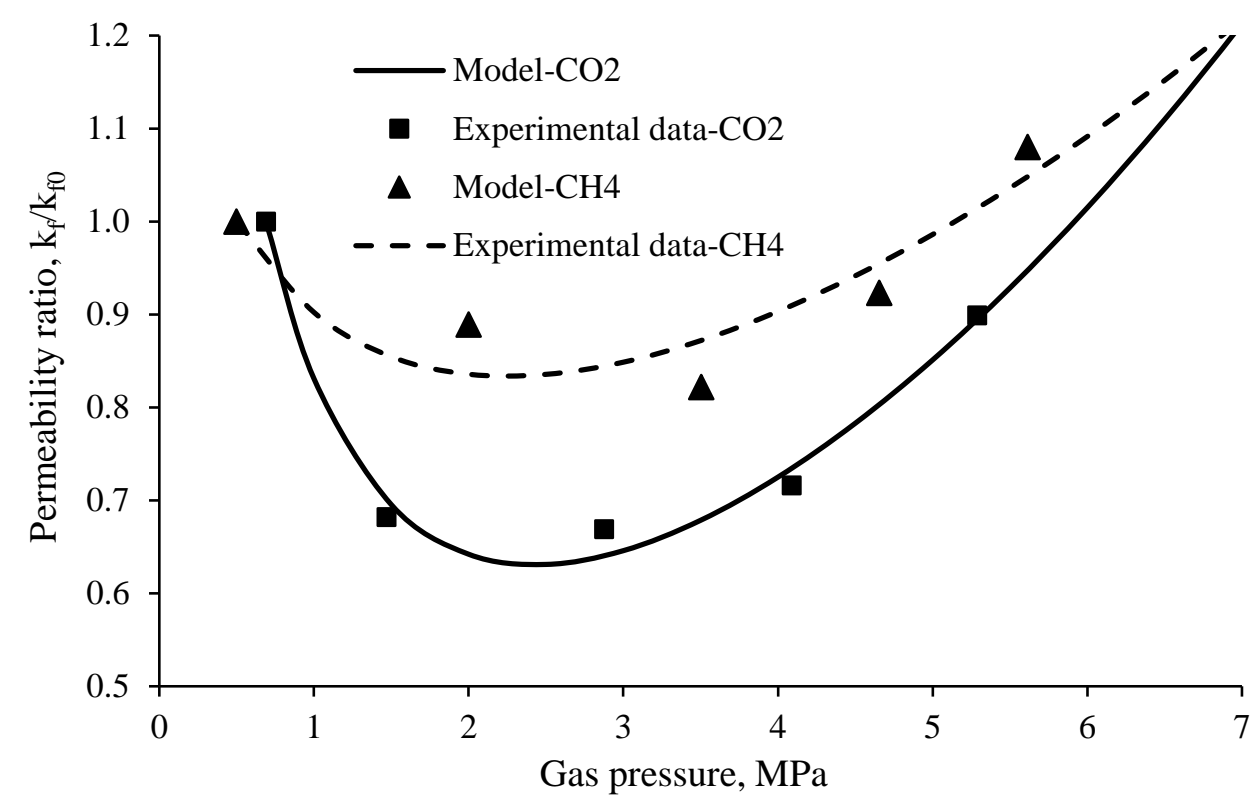

Fig. 7 Comparison of model with experimental data published by Robertson (2005).

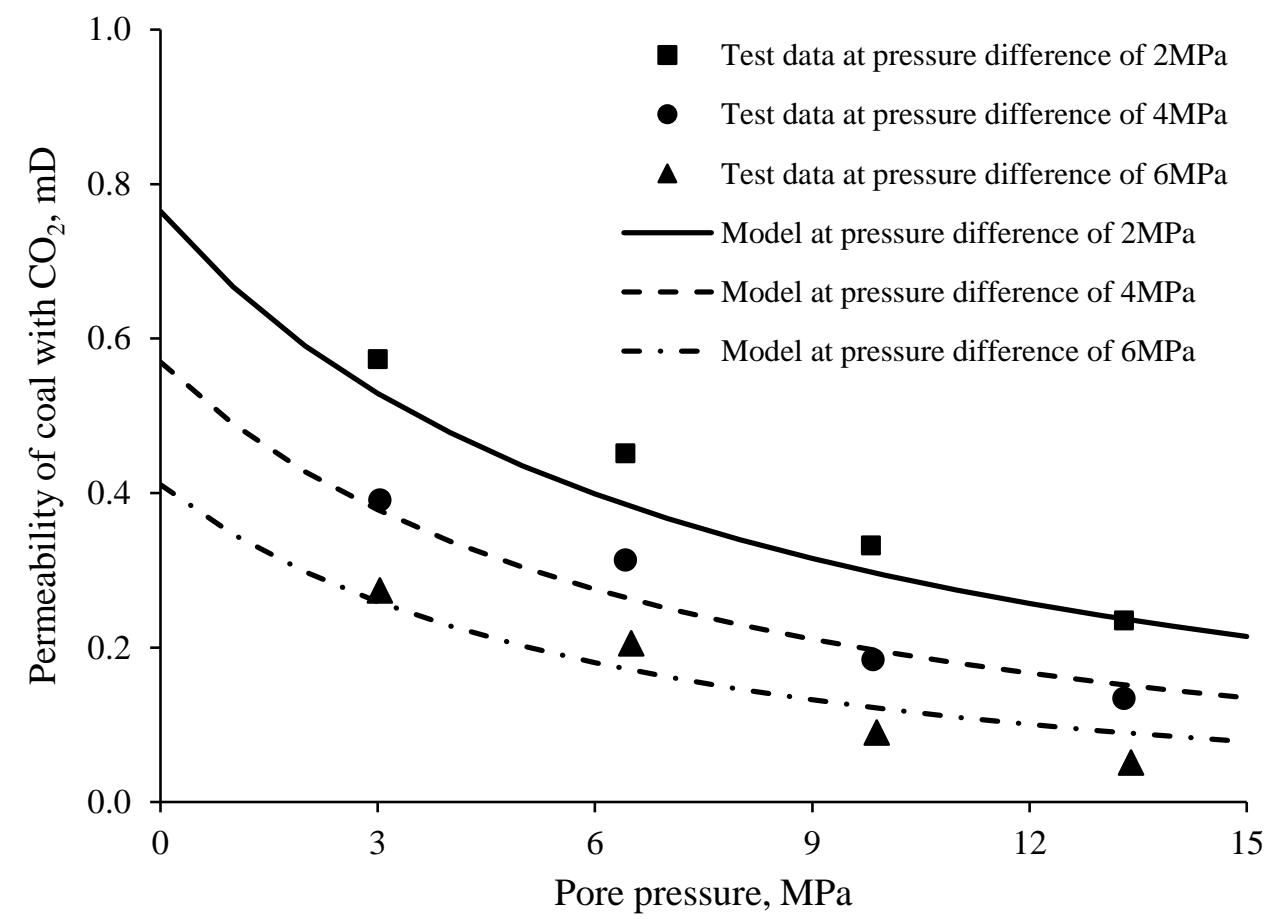

Fig. 8 Comparison of the model predictions with the test data for permeability of coal with $\mathrm{CO}_{2}$ versus pore pressures at different differential pressure from Pan et al (2010). 


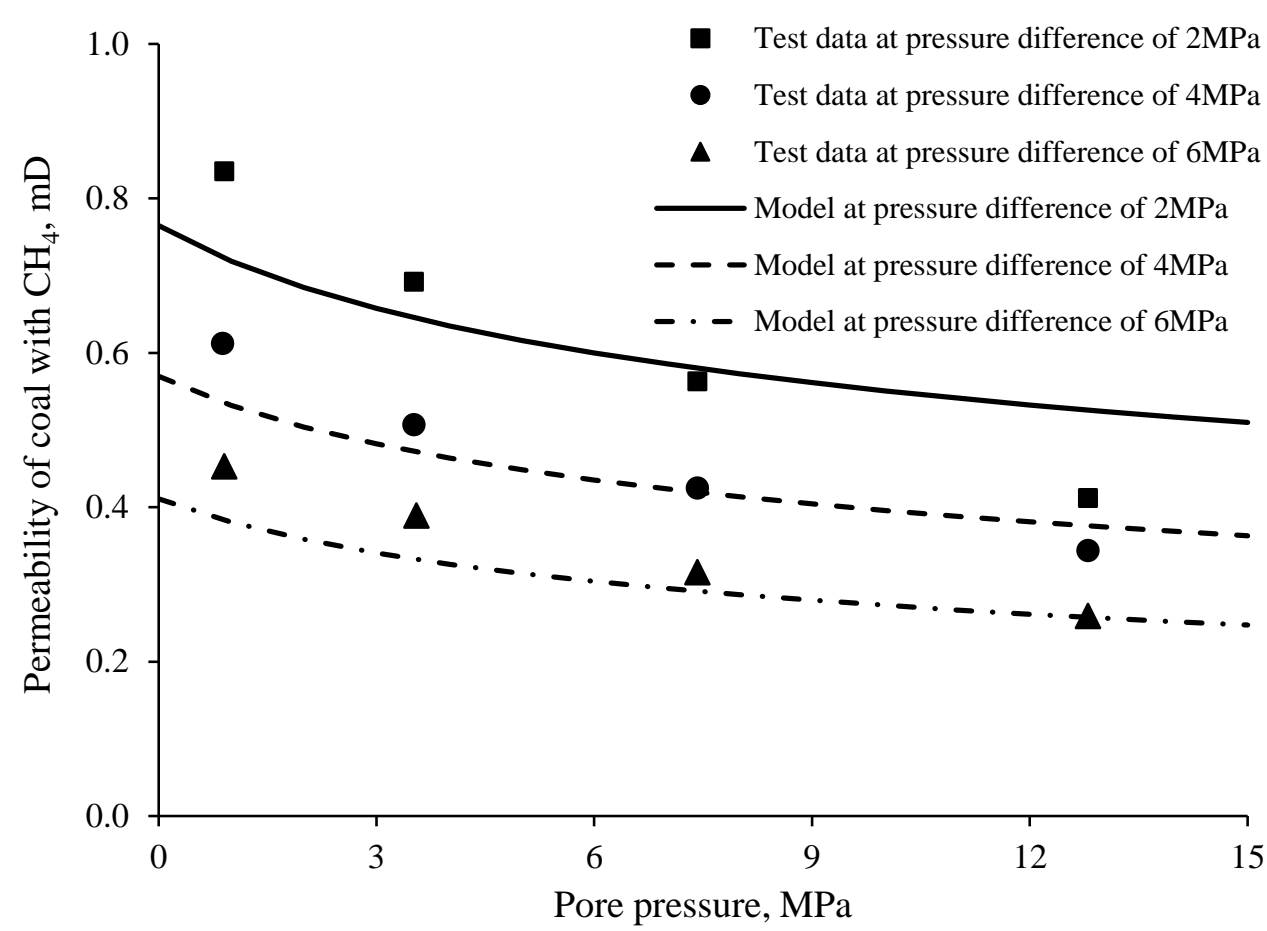

Fig.9 Comparison of the model predictions with the test data for permeability of coal with $\mathrm{CH}_{4}$ versus pore pressures at different differential pressures from Pan et al (2010).

Fig. 7 shows the data match between model results and experimental data provided by Robertson (2005). Under conditions of constant confining stress and with increasing pore pressure, the permeability measured with $\mathrm{CO}_{2}$ first drops by a factor of $33 \%$ with an increase in pore pressure to $2.5 \mathrm{MPa}$ (due to swelling), and then increases to roughly its original value at a pore pressure of $5.4 \mathrm{MPa}$ (due to poromechanical effect). Permeability decline with $\mathrm{CH}_{4}$ is not as significant compared to the permeability results measured by $\mathrm{CO}_{2}$. Good matches are obtained between the model results and the laboratory data. The permeability calculated using the newly developed model verses the $\mathrm{CO}_{2}$ and $\mathrm{CH}_{4}$ pore pressure under differential pressures of $2 \mathrm{MPa}, 4 \mathrm{MPa}$ and $6 \mathrm{MPa}$ is also compared with the permeability measurements by Pan et al. (2010), as shown in Fig. 8 and Fig. 9. It can be seen from Fig. 8 and Fig. 9 that the permeability model developed in this work can reliably predict the permeability. Larger deviations from the test data are observed only in the range of lower pore pressure for $\mathrm{CH}_{4}$ permeability in Fig. 9, where the model prediction overestimates the corresponding measurements. Both sets of data matches indicate the validity of the newly-developed coal permeability model, providing further confidence on the accuracy of the theoretical formulation for permeability implemented in the numerical model and the reasonability of the internal interaction coefficient. 


\section{Simulation and discussion}

In this section, a series of numerical simulations are performed to investigate the impact of adsorption on flow behaviour during $\mathrm{CO}_{2}$ injection into a coal core typical of those tested at the laboratory scale. A cylindrical geometry (100 $\mathrm{mm}$ high and $50 \mathrm{~mm}$ wide) confined by constant stress is selected for the numerical model, as shown in Fig. 10a. Due to the symmetry, the cylinder coal sample can be simplified as the 2D model pictured in Fig. 10b.

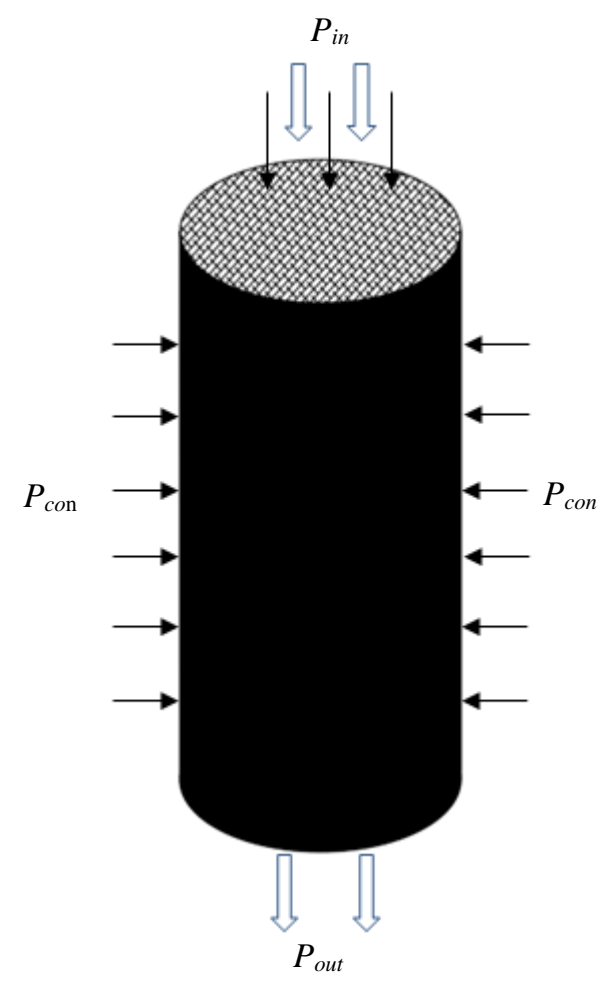

(a) Laboratory coal sample

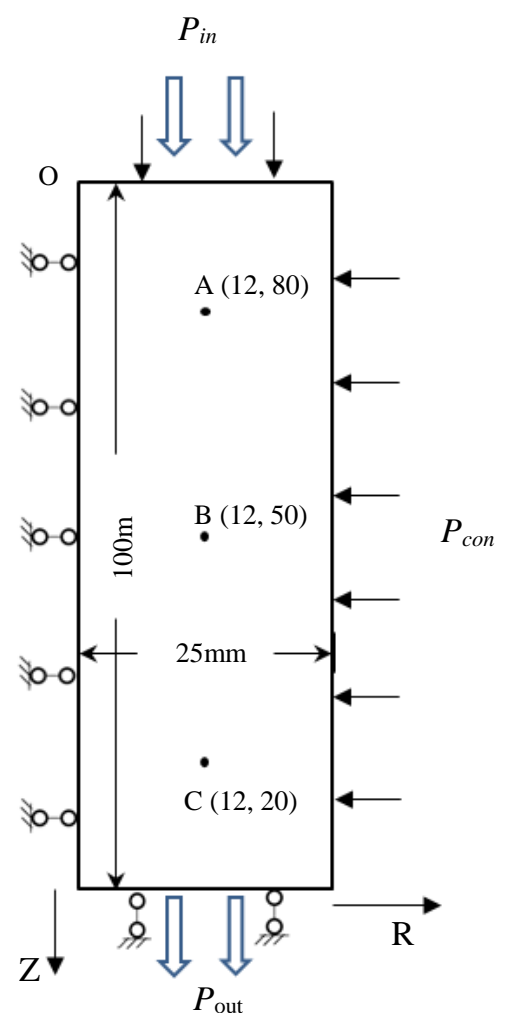

(b) 2-D model

Fig. 10 Geometry and boundary conditions of the numerical model under constant confining stress.

Boundary conditions corresponding to the conditions shown in Fig. 10 are required for this coupled problem. For coal deformation, a vertical constraint is applied to the outflow boundary while a constant confining stress is applied to the right and inflow boundaries. The left side of the model is fixed horizontally. For gas flow, a zero flux boundary is applied to the right and left sides of model. For gas flow in the matrix continuum, the zero flux conditions are specified for each boundary of the model. The initial pressure for both fracture and matrix continua is $0.1 \mathrm{MPa}$. Three measuring points with an equal spacing of $30 \mathrm{~mm}$ are set to monitor the variations of the gas pressures and permeability, i.e. $\mathrm{A}(12,80), \mathrm{B}(12,50)$ and $\mathrm{C}(12,20)$. The parameters for simulations are listed in Table 4, some of which are chosen from the validation tests presented above, with others selected from the literature (Gensterblum et al., 2010; Peng et al., 2014; Zhang et al., 2018). In this simulation, the selected fracture porosity is higher than the matrix porosity. This is taken as reasonable since Robertson and Christiansen (2006) present a photograph of the coal sample used, in which clearly-defined fractures can be seen. A time-dependent injection pressure is specified at the top surface as an inflow boundary to the fracture 
continuum for the gas transport model, a constant loading speed is assumed, and the characteristic time for equilibrium fracture pressures is set 2500 seconds.

Table 4 Parameters input in the numerical simulation.

\begin{tabular}{|l|l|l|}
\hline Parameters & Value & Reference \\
\hline Bulk modulus of coal, $K, \mathrm{GPa}$ & 1.1 & \\
\hline Modulus of coal matrix, $K_{m}, \mathrm{GPa}$ & 2.8 & Robertson (2005) \\
\hline Modulus of solid grains, $K_{s}, \mathrm{GPa}$ & 3.6 & \\
\hline Poisson's ratio, $v$ & 0.35 & Peng et al. (2014) \\
\hline Initial porosity of fracture, $n_{f 0}$ & 0.013 & Peng et al. (2014) \\
\hline Initial porosity of matrix, $n_{m 0}$ & 0.01 & Peng et al. (2014) \\
\hline Initial fracture permeability, $k_{f 0}, \mathrm{~m}^{2}$ & $8.5 \mathrm{e}-14$ & Zhang et al. (2018) \\
\hline Density of coal matrix, $\rho_{c}, \mathrm{~kg} \mathrm{~m}^{-3}$ & 1400 & \\
\hline Density of gas at standard condition, $\rho_{s}, \mathrm{~kg} \mathrm{~m}^{-3}$ & 1.78 & Zhang et al. (2018) \\
\hline Viscosity of gas, $\mu, \mathrm{Pa} \mathrm{s}$ & $1.84 \mathrm{e}-5$ & Peng et al.(2014) \\
\hline Langmuir volume constant, $V_{\mathrm{L}}, \mathrm{m}^{3} \mathrm{~kg}^{-1}$ & 0.035 & Gensterblum et al. (2010) \\
\hline Langmuir pressure constant, $B, \mathrm{MPa}^{-1}$ & 0.88 & \\
\hline Material parameter, $\varphi_{f}, \mathrm{~mol} \mathrm{~m}{ }^{-3}$ & 0 & \\
\hline Material parameter, $\varphi_{m}, \mathrm{~mol} \mathrm{~m} \mathrm{~m}^{-3}$ & $0.16 \mathrm{e} 6$ & \\
\hline Temperature, $T, \mathrm{~K}$ & 300 & Robertson (2005) \\
\hline Interaction change rate, $\omega, \mathrm{MPa}^{-1}$ & $7.4 \mathrm{e}-4$ & \\
\hline Initial interaction coefficient, $\chi_{0}, \mathrm{MPa}^{-1}$ & $3.5 \mathrm{e}-5$ & \\
\hline Sorption time, $\tau, \mathrm{s}$ & $2.0 \mathrm{e} 5$ & Peng et al. (2014) \\
\hline
\end{tabular}

Fig. 11 depicts the distribution of the gas pressure in the fracture and matrix continua along the coal sample after the equilibrium state is reached. The high pressure is located at the gas inlet of the coal sample, deceasing gradually to the gas outlet pressure. For the matrix porosity, the high-pressure area shows larger matrix porosity. The matrix porosity increases from $1 \%$ to about $7 \%$ with the increase of gas pressure from $0.7 \mathrm{MPa}$ to $7 \mathrm{MPa}$. However, the increase of matrix porosity tends to be slow with increase in the gas pressure. The fracture porosity displays a different pattern from the matrix porosity. The fracture porosity across most of the core is less than the initial value and decreases to the minimum at the location where the pressure is approximately $2.2 \mathrm{MPa}$. 


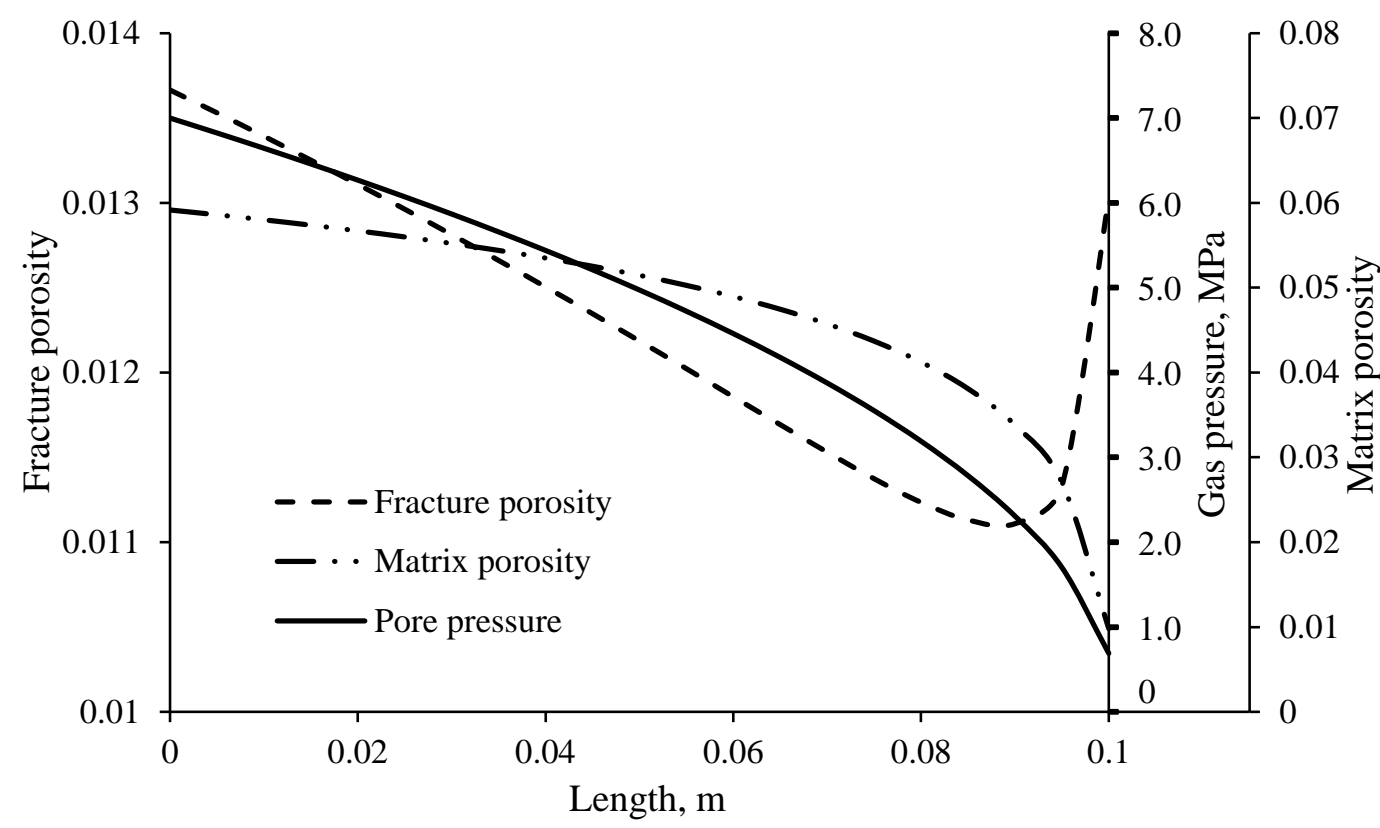

Fig.11 Spatial distribution of the gas pressure and porosities in the coal sample under equilibrium state.

In order to better understand the variation of gas pressures in the fracture and matrix continua with equilibrium time, three monitoring points are set to explore the characteristics of gas flow. Due to the larger permeability of the fracture continuum, the fracture gas pressure rapidly increases and reaches the equilibrium state almost at the same time. The point closer to the gas inlet of the coal sample has higher gas pressures as shown in Fig. 12. It can be seen that almost no gas diffuses into the coal matrix before the fracture gas pressure achieves equilibrium. There is a rapid increase in matrix gas pressure after equilibrium of the fracture gas pressure. However, the equilibrium time for gas pressure in the matrix at these three monitoring points is different. The equilibrium time for point $\mathrm{A}$ is relatively shorter than the other two points, as expected since higher pressures in the fracture continuum promote gas diffusion into coal matrix continuum and reduce the equilibrium time. 


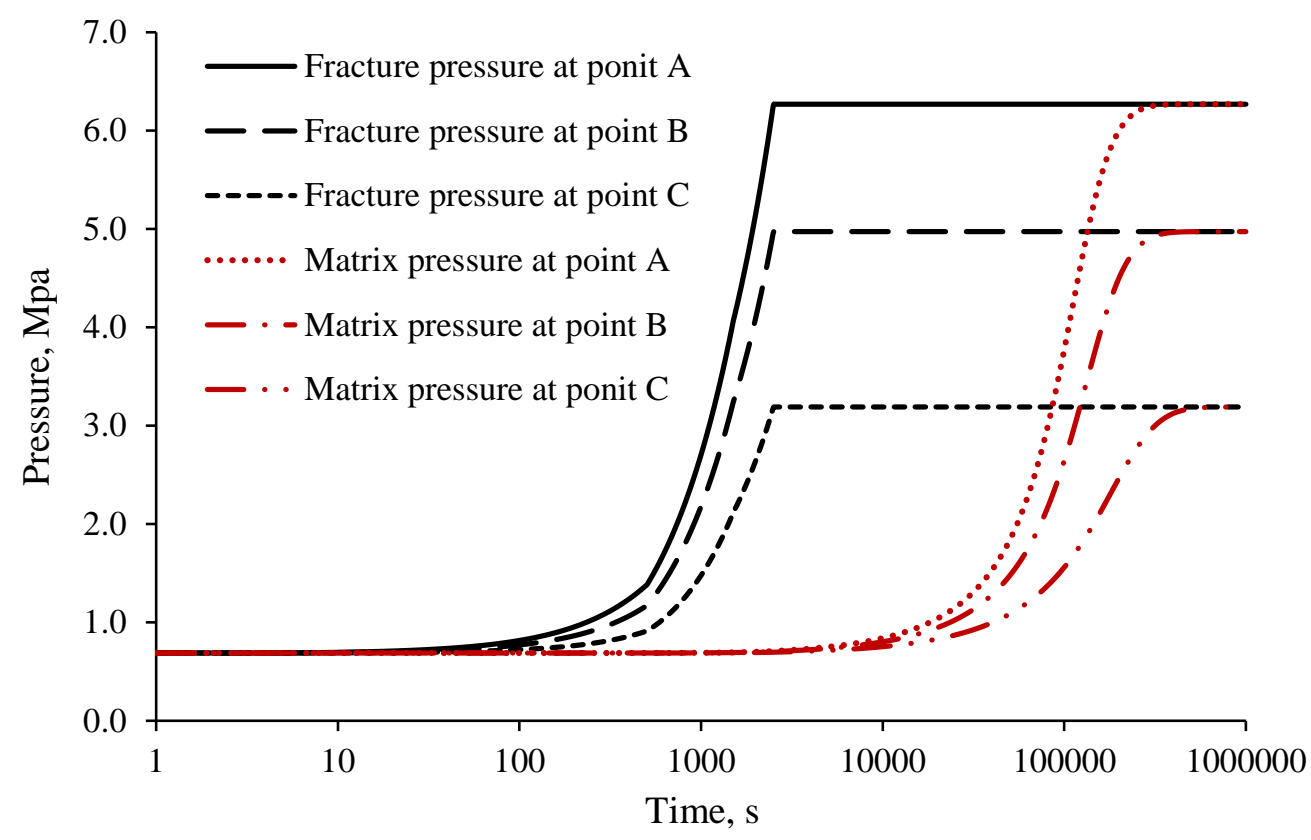

Fig. 12 The evolution of gas pressures in fracture and matrix with time at three detection points.

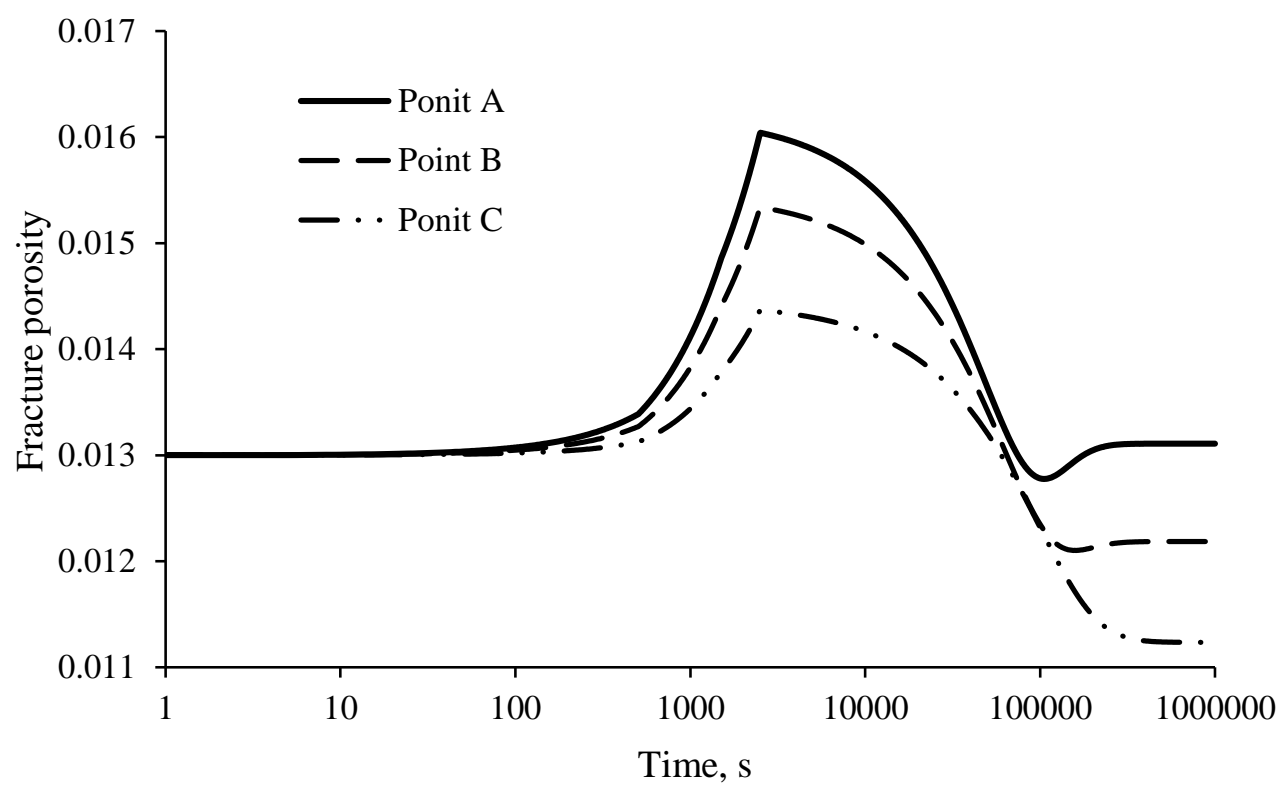

Fig.13 The evolution of fracture porosity with time at three detection points. 


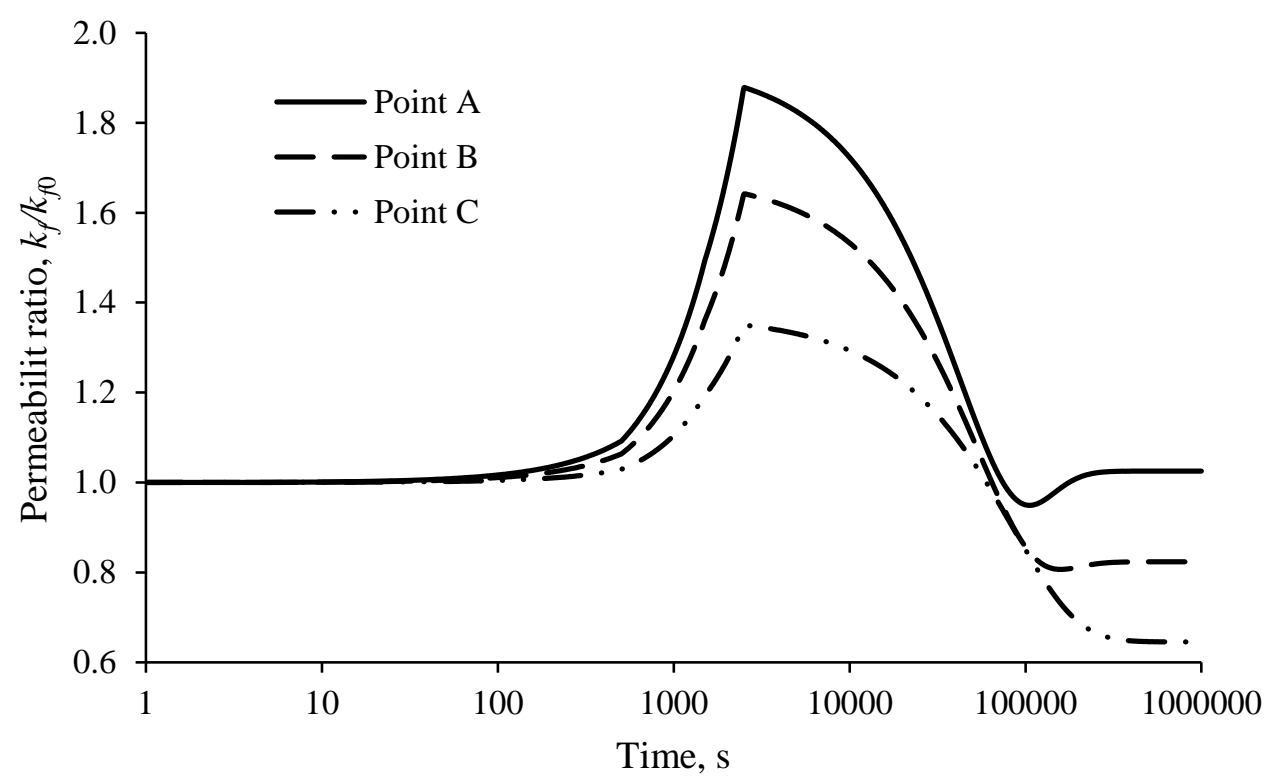

Fig. 14 The evolution of fracture permeability with time at three detection points.

The overall relationships between the simulation time and the evolving coal porosity and permeability are illustrated in Fig. 13-15. Fig. 13 shows that the patterns of porosity variation with time at the three monitoring points are similar at early simulation times; the rapid increase in fracture gas pressure results in the decrease of effective stress and increase of fracture porosity, and fracture porosity change is controlled by the first two terms in equation (27a). Mass exchange between the fracture continuum and coal matrix continuum occurs with the increase in simulation time, leading to an increase in the gas pressure of the coal matrix continuum and therefore the amount of gas adsorption. In turn, the increase of the amount of adsorbed gas induces the decrease in surface stress at the fluid-solid interface, leading to the increase in pressure difference between the coal matrix and fracture system. The change in fracture volume due to internal deformation of the coal is competitive with that due to the bulk volume change induced by effective stress; that is, the third term in equation (27a) plays a controlled role in the variation of fracture porosity. The volume occupied by the fractures is compressed. Therefore, the fracture porosity declines during this period of the simulation. The fracture porosity of point A shows an obvious rebound before reaching equilibrium compared with these of points $\mathrm{B}$ and $\mathrm{C}$. This is because with the increase in gas pressure in the matrix continuum as a result of continuous diffusion of gas into coal matrix, the change in fracture volume due to the internal deformation of coal is smaller than the change in bulk volume induced by effective stress; the fracture porosity is mainly affected by the effective stress when the gas pressure is larger. The pressures at points B and C are relatively lower and the impact of internal interactions on changes to the fracture volume are still dominant, and thus no rebounds are displayed. The permeability evolutions with equilibrium time at three detection points show the same trend as fracture porosity, as shown in Fig. 14. 


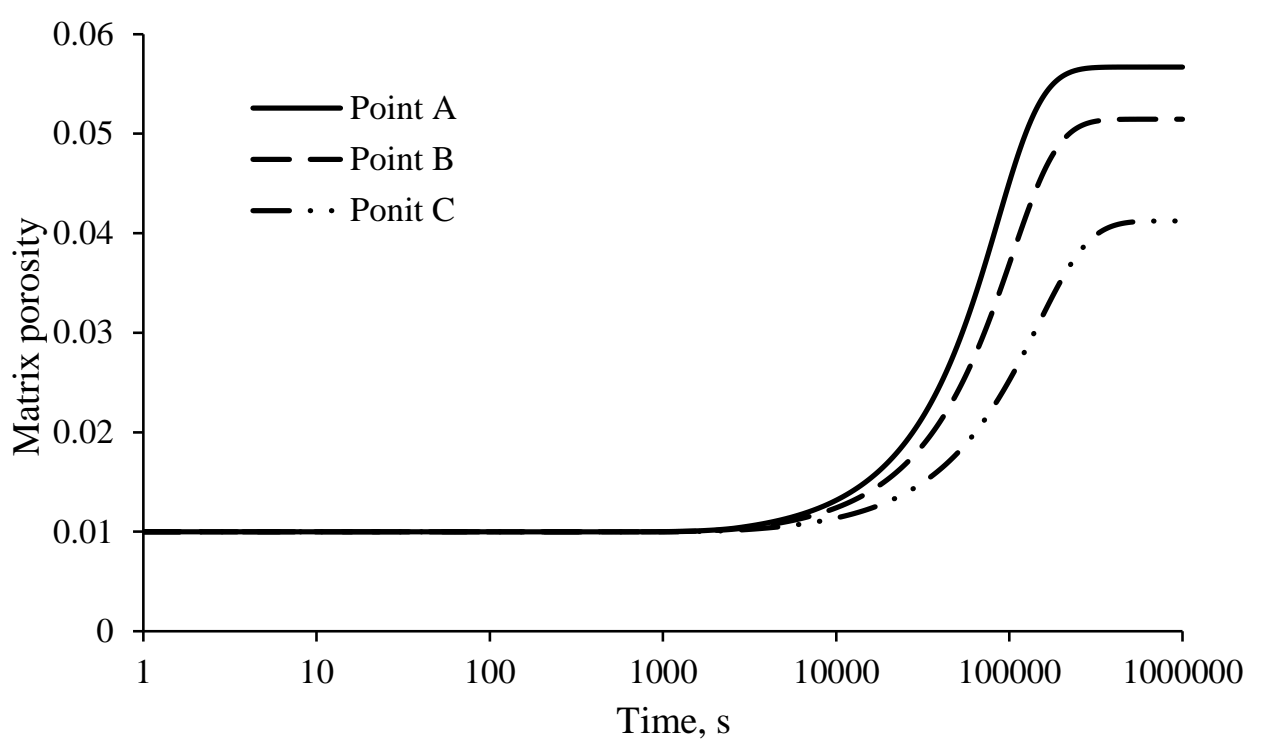

Fig. 15 The evolution of matrix porosity with time at three detection points.

In previous studies, most of the focus has fallen on the fracture porosity and permeability; attempts to consider the variation of matrix porosity are limited. Fig. 15 depicts how the matrix porosity varies with equilibrium time. Compared with the fracture porosity, $\mathrm{CO}_{2}$ injection into coal has the potential to increase the microporosity significantly, similar findings have been reported in other literature (Gathitu et al., 2009; Liu et al., 2010). For example, the matrix porosity at point A increases from the initial porosity of $1 \%$ to about $5.5 \%$ at equilibrium state. It can be seen that the matrix porosity shows a similar pattern to the gas pressure in the matrix, illustrated in Fig. 12. At the early stage, although the bulk gas pressure in the fracture network is higher than that in matrix system and matrix pores can be contracted, the increase in bulk volume caused by bulk gas pressure in the fracture network can bring about an increase in micropore volume of the coal matrix at the same time. Thus, the porosity does not show an obvious decrease during the early period. With time increase, the bulk gas pressure in the matrix increases as a consequence of more gas diffusion into the coal matrix from the fracture network, leading to more gas being adsorbed onto internal walls of the coal and the larger influence of surface stress. Not only the increase of bulk volume and compression of solid constituent, but also the internal deformation makes contributions to changes in matrix porosity; in other words, the three terms on the right hand side of equation (27b) have positive effects on the matrix porosity. Compared with the volumetric strain (three times the longitudinal strain) illustrated in Fig. 5, it is interesting that the significant increase in microporosity seems to be an important reason for the occurrence of the swelling phenomenon during $\mathrm{CO}_{2}$ injection into coal. 


\section{Conclusions}

In this study, a fully-coupled dual poroelastic model for gas flow, adsorption and coal deformation has been developed based on an existing dual porosity numerical model of multiphase, multicomponent chemical-gas transport. The surface stress of the fluid-solid interface modified by adsorption behaviour has been conceptualized as two pre-stresses acting in the cleat system and coal matrix, respectively, which facilitates the link between adsorption behaviour and mechanical behaviour. The interactions between gas flow and coal deformation have been integrated into this general porosity model, in which the fracture-matrix compartment interaction is taken into account by proposing a pressure differencedependent internal interaction coefficient. The model has been tested against analytical solutions and experiment measurements obtained from the literature; building on these works, the model developed in this study provides a capacity to predict the evolution characteristics of pressure and porosity in the fracture system and coal matrix during adsorption. The major observations from this work include:

(1) When the equilibrium state is reached, the high pressure is located at the area close to the inlet of the coal sample, deceasing gradually to the gas outlet pressure. The high-pressure area shows larger matrix porosity, while the increase of matrix porosity tends to be slow with the increase in gas pressure. The fracture porosity over much of the coal core is lower than the initial value and decreases to the minimum at locations close to the outlet.

(2) The fracture gas pressure rapidly increases and reaches the equilibrium state almost at the same time across the length of the coal sample. However, a rapid increase in matrix gas pressure occurs only after equilibrium of the fracture gas pressure. Also, high pressure in the fracture continuum enhances gas diffusion into the coal matrix continuum and reduces the equilibrium time.

(3) The variation of fracture porosity is relatively complex as a result of competing effective stress and internal deformation. At the initial stage, the fracture porosity increases due to the decrease in effective stress. As the free gas in the fracture continuum diffuses into the coal matrix continuum, the internal deformation becomes larger and compresses the fracture system, resulting in a decrease in fracture porosity. As the pore pressure increases, the change in the fracture volume due to bulk volume changes induced by effective stress is greater than that due to internal deformation; hence, the fracture porosity increases again.

(4) The matrix porosity shows a similar change trend to the matrix gas pressure and increases significantly. Compared to the fracture porosity, it is found that $\mathrm{CO}_{2}$ injection in coal has the potential to increase significantly the microporosity of coal, which seems to be an important reason for coal swelling and, contrary to the reduced fracture porosity, is beneficial for $\mathrm{CO}_{2}$ storage. 


\section{Acknowledgements}

The financial support provided by the China Scholarship Council for the Ph.D. studentship of the first author is gratefully acknowledged. The financial support provided by the Welsh European Funding Office (WEFO), through the FLEXIS project, is also gratefully acknowledged.

\section{References}

Borja, R.I., Koliji, A., 2009. On the effective stress in unsaturated porous continua with double porosity. Journal of the Mechanics and Physics of Solids 57, 1182-1193.

Chen, Z., Liu, J., Pan, Z., Connell, L.D., Elsworth, D., 2012. Influence of the effective stress coefficient and sorption-induced strain on the evolution of coal permeability: model development and analysis. International Journal of Greenhouse Gas Control 8, 101-110.

Connell, L.D., Lu, M., Pan, Z., 2010. An analytical coal permeability model for tri-axial strain and stress conditions. International Journal of Coal Geology 84, 103-114.

Cui, X., Bustin, R.M., 2005. Volumetric strain associated with methane desorption and its impact on coalbed gas production from deep coal seams. Aapg Bulletin 89, 1181-1202.

Day, S., Fry, R., Sakurovs, R., 2011. Swelling of moist coal in carbon dioxide and methane. International Journal of Coal Geology 86, 197-203.

Detournay, E., Cheng, A., 1993. Fundamentals of poroelasticity, comprehensive rock engineering: principles, practice and projects. Analysis and design method. Pergamon Press, Oxford.

Espinoza, D., Vandamme, M., Pereira, J.-M., Dangla, P., Vidal-Gilbert, S., 2014. Measurement and modeling of adsorptive-poromechanical properties of bituminous coal cores exposed to $\mathrm{CO}_{2}$ : Adsorption, swelling strains, swelling stresses and impact on fracture permeability. International Journal of Coal Geology 134, 80-95.

Fujioka, M., Yamaguchi, S., Nako, M., 2010. $\mathrm{CO}_{2}$-ECBM field tests in the Ishikari Coal Basin of Japan. International Journal of Coal Geology 82, 287-298.

Gathitu, B.B., Chen, W.-Y., McClure, M., 2009. Effects of coal interaction with supercritical $\mathrm{CO}_{2}$ : physical structure. Industrial \& Engineering Chemistry Research 48, 5024-5034.

Gensterblum, Y., Van Hemert, P., Billemont, P., Battistutta, E., Busch, A., Krooss, B., De Weireld, G., Wolf, K.-H., 2010. European inter-laboratory comparison of high pressure $\mathrm{CO}_{2}$ sorption isotherms II: Natural coals. International Journal of Coal Geology 84, 115-124.

Hol, S., Spiers, C.J., 2012. Competition between adsorption-induced swelling and elastic compression of coal at $\mathrm{CO}_{2}$ pressures up to $100 \mathrm{MPa}$. Journal of the Mechanics and Physics of Solids 60, 18621882.

Hosking, L.J., Thomas, H.R., Sedighi, M., 2017. A dual porosity model of high-pressure gas flow for geoenergy applications. Canadian Geotechnical Journal 55, 839-851. 
Kramer, D., Weissmüller, J., 2007. A note on surface stress and surface tension and their interrelation via Shuttleworth's equation and the Lippmann equation. Surface Science 601, 30423051.

Lewis, R., Pao, W., 2002. Numerical simulation of three-phase flow in deforming fractured reservoirs. Oil \& Gas Science and Technology 57, 499-514.

Liu, C., Wang, G., Sang, S., Rudolph, V., 2010. Changes in pore structure of anthracite coal associated with $\mathrm{CO}_{2}$ sequestration process. Fuel 89, 2665-2672.

Liu, H.-H., Rutqvist, J., 2010. A new coal-permeability model: internal swelling stress and fracturematrix interaction. Transport in Porous Media 82, 157-171.

Liu, J., Chen, Z., Elsworth, D., Miao, X., Mao, X., 2011a. Evolution of coal permeability from stresscontrolled to displacement-controlled swelling conditions. Fuel 90, 2987-2997.

Liu, J., Chen, Z., Elsworth, D., Qu, H., Chen, D., 2011b. Interactions of multiple processes during CBM extraction: a critical review. International Journal of Coal Geology 87, 175-189.

Liu, J., Wang, J., Chen, Z., Wang, S., Elsworth, D., Jiang, Y., 2011c. Impact of transition from local swelling to macro swelling on the evolution of coal permeability. International Journal of Coal Geology 88, 31-40.

Liu, Q., Cheng, Y., Zhou, H., Guo, P., An, F., Chen, H., 2015. A mathematical model of coupled gas flow and coal deformation with gas diffusion and Klinkenberg effects. Rock Mechanics and Rock Engineering 48, 1163-1180.

Liu, T., Lin, B., Yang, W., 2017. Impact of matrix-fracture interactions on coal permeability: model development and analysis. Fuel 207, 522-532.

Minkoff, S.E., Stone, C.M., Bryant, S., Peszynska, M., Wheeler, M.F., 2003. Coupled fluid flow and geomechanical deformation modeling. Journal of Petroleum Science and Engineering 38, 37-56. Myers, A., Prausnitz, J.M., 1965. Thermodynamics of mixed - gas adsorption. AlChE journal 11, 121127.

Nikoosokhan, S., Brochard, L., Vandamme, M., Dangla, P., Pellenq, R.J.M., Lecampion, B., Fen-Chong, T., 2013. $\mathrm{CO}_{2}$ Storage in Coal Seams: Coupling Surface Adsorption and Strain. 115-132.

Nikoosokhan, S., Brochard, L., Vandamme, M., Dangla, P., Pellenq, R.J.M., Lecampion, B., Fen Chong, T., 2012. $\mathrm{CO}_{2}$ Storage in Coal Seams: Coupling Surface Adsorption and Strain. Geomechanics in CO Storage Facilities, 115-132.

Oudinot, A.Y., Koperna, G.J., Philip, Z.G., Liu, N., Heath, J.E., Wells, A., Young, G.B., Wilson, T., 2011. $\mathrm{CO}_{2}$ injection performance in the Fruitland Coal Fairway, San Juan Basin: results of a field pilot. SPE Journal 16, 864-879.

Pan, Z., Connell, L.D., 2007. A theoretical model for gas adsorption-induced coal swelling. International Journal of Coal Geology 69, 243-252. 
Pan, Z., Connell, L.D., Camilleri, M., 2010. Laboratory characterisation of coal reservoir permeability for primary and enhanced coalbed methane recovery. International Journal of Coal Geology 82, 252261.

Pao, W.K., Lewis, R.W., 2002. Three-dimensional finite element simulation of three-phase flow in a deforming fissured reservoir. Computer methods in applied mechanics and engineering 191, 26312659.

Peng, Y., Liu, J., Wei, M., Pan, Z., Connell, L.D., 2014. Why coal permeability changes under free swellings: New insights. International Journal of Coal Geology 133, 35-46.

Pini, R., Ottiger, S., Burlini, L., Storti, G., Mazzotti, M., 2009. Role of adsorption and swelling on the dynamics of gas injection in coal. Journal of Geophysical Research: Solid Earth 114.

Reeves, S., Taillefert, A., Pekot, L., Clarkson, C., 2003. The Allison unit $\mathrm{CO}_{2}-\mathrm{ECBM}$ pilot: a reservoir modeling study. Topical Report, US Department of Energy. DE-FC26-0NT40924.

Robertson, E.P., 2005. Measurement and modeling of sorption-induced strain and permeability changes in coal. Idaho National Laboratory (INL).

Robertson, E.P., Christiansen, R.L., 2006. A permeability model for coal and other fractured, sorptiveelastic media. Idaho National Laboratory (INL).

Sampath, K., Perera, M., Ranjith, P., Matthai, S., Rathnaweera, T., Zhang, G., Tao, X., 2017. $\mathrm{CH}_{4}-\mathrm{CO}_{2}$ gas exchange and supercritical $\mathrm{CO}_{2}$ based hydraulic fracturing as $\mathrm{CBM}$ production-accelerating techniques: A review. Journal of $\mathrm{CO}_{2}$ Utilization 22, 212-230.

Seetharam, S., Thomas, H., Cleall, P.J., 2007. Coupled thermo/hydro/chemical/mechanical model for unsaturated soils-Numerical algorithm. International Journal for Numerical Methods in Engineering 70, 1480-1511.

Shi, J., Durucan, S., 2005. $\mathrm{CO}_{2}$ storage in deep unminable coal seams. Oil \& gas science and technology 60, 547-558.

Thomas, H.R., He, Y., 1998. Modelling the behaviour of unsaturated soil using an elastoplastic constitutive model. Géotechnique 48, 589-603.

Thomas, H.R., Sedighi, M., Vardon, P.J., 2012. Diffusive reactive transport of multicomponent chemicals under coupled thermal, hydraulic, chemical and mechanical conditions. Geotechnical and Geological Engineering 30, 841-857.

van Bergen, F., Pagnier, H., Krzystolik, P., 2006. Field experiment of enhanced coalbed methane- $\mathrm{CO}_{2}$ in the upper Silesian basin of Poland. Environmental Geosciences 13, 201-224.

Vandamme, M., Brochard, L., Lecampion, B., Coussy, O., 2010. Adsorption and strain: The $\mathrm{CO}_{2}-$ induced swelling of coal. Journal of the Mechanics and Physics of Solids 58, 1489-1505.

Warren, J., Root, P.J., 1963. The behavior of naturally fractured reservoirs. Society of Petroleum Engineers Journal 3, 245-255. 
White, C.M., Smith, D.H., Jones, K.L., Goodman, A.L., Jikich, S.A., LaCount, R.B., DuBose, S.B., Ozdemir, E., Morsi, B.I., Schroeder, K.T., 2005. Sequestration of carbon dioxide in coal with enhanced coalbed methane recovery a review. Energy \& Fuels 19, 659-724.

Wong, S., Law, D., Deng, X., Robinson, J., Kadatz, B., Gunter, W.D., Jianping, Y., Sanli, F., Zhiqiang, F., 2007. Enhanced coalbed methane and $\mathrm{CO} 2$ storage in anthracitic coals-micro-pilot test at South Qinshui, Shanxi, China. International Journal of Greenhouse Gas Control 1, 215-222.

Wu, Y.-S., Li, J., Ding, D., Wang, C., Di, Y., 2014. A generalized framework model for the simulation of gas production in unconventional gas reservoirs. SPE Journal 19, 845-857.

Zhang, S., Liu, J., Wei, M., Elsworth, D., 2018. Coal permeability maps under the influence of multiple coupled processes. International Journal of Coal Geology.

Zhou, Y., Li, Z., Yang, Y., Zhang, L., Qi, Q., Si, L., Li, J., 2016. Improved porosity and permeability models with coal matrix block deformation effect. Rock Mechanics and Rock Engineering 49, 36873697. 